\title{
COMPETITIVE ATHLETES' EXPERIENCES WITH MENTAL ILLNESS AND PSYCHOLOGICAL TREATMENT
}

\author{
by \\ Rachel Jewett \\ Master of Science, University of Toronto, 2017 \\ Bachelor of Kinesiology, University of Toronto, 2015 \\ A thesis presented to Ryerson University \\ in partial fulfillment of the \\ requirements for the degree of \\ Master of Arts in the program of Psychology
}

Toronto, Ontario, Canada, 2019

(C) Rachel Jewett, 2019 


\section{AUTHOR'S DECLARATION FOR ELECTRONIC SUBMISSION OF A THESIS}

I hereby declare that I am the sole author of this thesis. This is a true copy of the thesis, including any required final revisions, as accepted by my examiners.

I authorize Ryerson University to lend this thesis to other institutions or individuals for the purpose of scholarly research.

I further authorize Ryerson University to reproduce this thesis by photocopying or by other means, in total or in part, at the request of other institutions or individuals for the purpose of scholarly research.

I understand that my thesis may be made electronically available to the public. 


\title{
COMPETITIVE ATHLETES' EXPERIENCES WITH MENTAL ILLNESS AND PSYCHOLOGICAL TREATMENT
}

\author{
Master of Arts, 2019 \\ Rachel Jewett \\ Psychology \\ Ryerson University
}

The purpose of the study was to explore the role of sport culture in athlete mental health, the importance of mental healthcare providers having an understanding of this context, and how athletes tend to access mental healthcare. Ten athletes with personal experience in these domains were interviewed and a thematic analysis was used. Athletes who attributed their mental health challenges to their sport participation thought it was important for their mental healthcare providers to have experience with sport. Normalization and validation of mental illness within the sport context and understanding competitive sport culture were identified as important. Facilitators of care access included a supportive cultural environment and influential individuals. Barriers included stigma and the financial cost of treatment. Coaches influenced athletes' attitudes toward help-seeking. Implications for assessment and treatment of mental illness in athletes are discussed. Future research should explore gender, race, and ethnicity in athletes' experiences with mental illness. 


\section{ACKNOWLEDGEMENTS}

I would like to acknowledge the critical role that my thesis supervisory committee, composed of Dr. Michelle Dionne and Dr. Maria Gurevich, played in the creation and implementation of this thesis. Thank you, Michelle, for your support in undertaking this project. The independence you have allowed me during this degree to pursue this research area is deeply appreciated. Maria, your guidance regarding the methodological approach for this project was extremely valuable.

Critical in this acknowledgement is the gratitude I must express to the athletes who participated in interviews and enthusiastically shared their stories and experiences with me and the readers of this work. Thank you for being vulnerable and forthcoming in sharing parts of your lives with me, I have learned a great deal from you about the challenges and joys of competitive sport.

Finally, thank you to my family, friends, and training partners for supporting me and giving me the inspiration and motivation to pursue this line of research. I am deeply grateful to have you in my life. 
Table of Contents

Abstract iii

Acknowledgements

List of Tables $\quad$ vi

List of Appendices vii

Purpose and Rationale $\quad 1$

Chapter 1: Literature Review 3

Mental Illness in Athletes and the Sport Context 3

Athletes' Perspectives on Effective Psychological Practitioners $\quad 8$

Access and Barriers to Mental Healthcare for Athletes 13

Conclusion $\quad 17$

$\begin{array}{lr}\text { Chapter 2: Methodology and Methods } & 18\end{array}$

Paradigmatic Perspective: Social Constructionism 19

Participant Inclusion Criteria $\quad 21$

Data Collection $\quad 21$

Participant recruitment $\quad 22$

$\begin{array}{ll}\text { Interviews } & 23\end{array}$

$\begin{array}{ll}\text { Reflexive journal } & 23\end{array}$

Data Analysis $\quad 24$

Chapter 3: Findings $\quad 26$

Chapter 4: Discussion $\quad 84$

Conclusions and Future Directions $\quad 97$

Appendices 102

Appendix A: Interview Guide 102

Appendix B: Mental Health Resources $\quad 104$

Appendix C: Recruitment Poster 108

References 109 


\section{LIST OF TABLES}

Table 1: Participant Characteristics

Table 2: Mental Healthcare Providers and Sport Knowledge

Table 3: Athletes' Venues of Mental Healthcare 


\section{LIST OF APPENDICES}

Appendix A: Interview Guide

102

Appendix B: Mental Health Resources

104

Appendix C: Recruitment Poster 
Competitive Athletes' Experiences with Mental Illness and Psychological Treatment

\section{Purpose and Rationale}

There is a widely held assumption that athletes are immune to psychopathology because of the supposed requirement to demonstrate mental toughness in training and competition (Schaal et al., 2011). In fact, many athletes do report symptoms of mental illness and present with clinical levels of many psychopathologies (Gulliver, Griffiths, Mackinnon, Batterham, \& Stanimirovic, 2015). Collegiate and elite athletes experience a range of mental illnesses at a rate similar to the general population, including depression, anxiety, eating disorders, attention-deficit hyperactivity disorder, personality disorders, and alcohol and substance abuse (Andersen, Denson, Brewer, \& Van Raalte, 1994; Green, Uryasz, Petr, \& Bray, 2001; Gulliver et al., 2015; Schaal et a., 2011; Yang et al., 2007). Notably, Gorczynski, Coyle and Gibson (2017) recently conducted a comparative meta-analysis and reported that elite athletes were just as likely to report depression symptoms as non-athletes.

At the same time, there are significant benefits derived from sport participation, such as enhanced self-esteem, social benefits, reduced stress and protection against depression later in life (Eime, Young, Harvey, Charity, \& Payne, 2013; Jewett et al., 2014), and collegiate athletes have reported that sport can serve as an important coping mechanism and source of reprieve from psychological stress (Jones, Butryn, Furst, \& Semerjian, 2013). At the same time, the athletes who Jones and colleagues (2013) interviewed reflected about how aspects of sport participation can also act as significant stressors. Researchers have suggested that due to the complex interaction of protective and stressful aspects of competitive sport participation, treatment for athletes who experience mental health challenges should be provided by clinicians who have specific experience with or knowledge of the sport (Glick \& Horsfall, 2009). While 
this claim seems intuitively appealing, the validity and practicality of it has not been specifically examined in the research literature. Furthermore, when considering the mental health and wellbeing of athletes and the most appropriate and effective ways to provide this population with mental healthcare, it would be worthwhile to explore athletes' perspectives of their mental health treatment experiences. Treatment efficacy in specific populations is typically investigated using randomized control trials and standardized measures of symptom changes (e.g. Jayasekara et al., 2015), while the perspective of treatment recipients is rarely considered. In the context of sport, the concept of mental health issues is intertwined with pervasive culturally produced discourses, such as the ideal of mental toughness, psychological challenges contributing to perceived weakness and vulnerability, and the performance narrative (Gucciardi, Hanton, \& Fleming, 2017; Douglas \& Carless, 2006). Given this connection between sport culture and athlete mental health, it would be useful to adopt qualitative approaches within a social constructionist paradigm. This paradigm allows for the broad and flexible exploration of how athletes' construct their perspectives of mental health and healthcare experiences as situated within the current social and cultural climate of competitive sport (Burr, 2015).

Therefore, the overarching purpose of the current inquiry is to generate knowledge to inform effective and appropriate treatment of athletes with mental illness and enhance athletes' access to mental healthcare. Specifically, the aim is to generate knowledge regarding the role of sport culture in the psychological stressors that athletes face, the importance athletes place on their mental healthcare provider having a specific understanding of this context, and the avenues by which athletes tend to access mental healthcare. Furthermore, these topics and how athletes' perspectives on these topics are understood in light of the prevailing discourses surrounding mental health in the sport context will be explored. 


\section{Chapter 1: Literature Review}

\section{Mental Illness in Athletes and the Sport Context}

Research has examined factors unique to the sport context that may affect the nature of mental health challenges in athletes. These factors include tangible stressors such as high physical injury rates and retirement from sport, as well as factors that are highly connected to the current cultural and social context of competitive sport such as the stigma of mental illness in athletes, competitive pressure, and high societal and internal performance expectations (Appaneal, Levine, Perna, \& Rohl, 2009; Douglas \& Carless, 2009; Gulliver, Griffiths, \& Mackinnon, 2012; Lavallee \& Robinson, 2007). Gulliver and colleagues (2012) conducted focus group interviews with 15 elite Australian athletes (average age of 19) about their perceptions of mental health issues affecting athletes. These athletes identified performance anxiety and depressed mood from poor performance as being significant stressors in addition to the following: sadness and isolation as a result of experiencing injury; being held to a high standard of behaviour by society; stress over weight control; difficulties balancing sport with other aspects of life such as family, work, or school; and the burden of often living far from family and friends. Older work with student-athlete populations identified many of the same challenges facing these athletes, such as identity conflict, fear of success or failure, social isolation, balancing sport and academic demands, injuries, drug and alcohol problems, and termination of collegiate athletic careers (Parham, 1993; Pinkerton, Hinz \& Barrow, 1989).

Experiencing injuries and the pain associated with them has consistently been associated with an increased risk of depression (Appaneal et al., 2009). Appaneal and colleagues (2009) administered self-report surveys and conducted clinical interviews with athletes post-injury and compared their responses with non-injured control subjects. The results of this study indicated 
that athletes with injuries had higher depression ratings compared to healthy athletes. Minimal research has specifically examined why injury may be associated with higher risk of depression but Smith and Milliner (1987) suggest that long rehabilitation times, inability to re-capture preinjury success, post-concussive syndrome, and stress over being replaced by teammates are significant stressors that injured athletes may face.

Retirement from a high-level of sport participation, such as elite and interuniversity levels, can also significantly impact athletes' psychological health (Lavallee \& Robinson, 2007; Wylleman, Alfermann, \& Lavallee, 2004). Athletes consistently report distress after retirement due to feelings of loss and helplessness, feelings that are particularly salient if an athlete's sense of identity is deeply entrenched in his or her sport participation (Jewett, Kerr, \& Tamminen, 2019; Kerr \& Dacyshyn, 2000; Lavallee \& Robinson, 2007; Lavallee, Gordon, \& Grove, 1997). Brewer, Van Raalte, and Linder's (1993) work on athletic identity led them to suggest that as athletes devote increasing amounts of their lives to training and competing, their self-concept may become restricted to the athletic sphere. It is understandable that retirement from the activity upon which a narrow self-identity has been built may represent a significant loss for such athletes. In addition, graduation and retirement from collegiate sport participation may not only pose a threat to an athlete's sense of identity but may also mean that an athlete no longer has easy access to support services such as physical and mental healthcare, as many athletes tend not to seek out resources beyond those offered within their athletic departments (Jewett et al., 2019; Pinkerton et al., 1989).

In addition to the more tangible stressors inherent in sport participation, such as injury and retirement, there are stressors that are related to the culture of competitive sport that contribute to internal and societal expectations of athletes that can be both motivating and 
psychologically damaging. Some of the cultural influences that play a role in how athletes experience mental health have been conceptualized as narratives or discourses, depending on the research approach from which they are written about. They are very similar concepts, essentially representing storylines or culturally-shared beliefs or meaning systems that exist within a particular culture and influence how individuals structure and understand their own and others' experiences (Burr, 2015; Smith, 2015). Elaborating on the discourses that are salient within sport culture and that are relevant to mental health, such as the performance narrative (Douglas \& Carless, 2006) and the desirability of mental toughness (Jones, Hanton, \& Connaughton, 2002), is important for being able to analyze how athletes perceive and thus talk about mental health and mental healthcare. These culturally produced concepts also come into play when discussing barriers to mental health help-seeking related to self- and other-perceived stigma.

The performance narrative is "a story of single-minded dedication to sport performance to the exclusion of other areas of life and self...winning, results, and achievements are preeminent and link closely to the storyteller's mental well-being, identity, and self-worth" (Douglas \& Carless, 2009, p. 3). This perspective within competitive sport encourages society and athletes to view winning as the only acceptable outcome of a sport performance. In addition, it encourages the expectation that it is to this end that an elite athlete should dedicate his or her life and anything less demonstrates lack of commitment and discipline. Informed by their longitudinal research with female professional golfers, Douglas and Carless $(2006,2009,2015)$ describe the performance narrative as the dominant perspective in elite sport and how it contributes to immense pressure to produce results and perform up to internal and external expectations, sometimes at the cost of athletes' mental health. These researchers also illustrate how the pressure to perform can leave athletes feeling as though they have limited options to 
develop other aspects of their lives and leave them vulnerable to extreme emotional turmoil over failure. Douglas and Carless (2009) caution about the repercussions for mental well-being, identity, and self-worth when winning, results, and achievement are the only priorities for athletes.

Another cultural narrative in competitive sport that may influence athletes' perspectives of mental health and healthcare is mental toughness, a concept that has become exceedingly popular as attention to mental performance and sport psychology has grown in recent years (Gucciardi, Hanton, \& Fleming, 2017). Many different definitions of mental toughness in sport have been proposed using various investigative approaches (e.g. Fourie \& Potgieter, 2001; Powell \& Myers, 2017; Sheard, Golby, \& van Wersch, 2009), however given our present focus on athletes' perspectives, I will draw on the work of Jones and colleagues (2002) to define the construct. These researchers conducted focus group interviews with ten international athletes to collectively establish the following definition of mental toughness:

The natural or developed psychological edge that enables you to generally cope better than your opponents with the many demands (competition, training, lifestyle) that sport places on a performer. Specifically, be more consistent and better than your opponents in remaining determined, focused, confident, and in control under pressure. (p. 209)

Mental toughness is largely considered a laudable attribute for an athlete to possess, in essence fundamental to achieving sport performance success. While distilling the somewhat amorphous and intangible psychological components of sport performance into the construct of "mental toughness" is understandably attractive, it is worth questioning the implications this terminology has for perceptions of mental health in sport. For example, it has been suggested that valorizing mental toughness contributes to stigma associated with mental illness in athletes (Bauman, 
2016). Through the lens of the discourse that mental toughness is necessary for success, it is a short leap to subsequently view mental illness as an inherent weakness, a vulnerability that may threaten the confidence that coaches, teammates, competitors, and even an athlete may have in his or her ability to perform. A dangerous repercussion of this discourse is that athletes may be extremely hesitant to seek help if they are experiencing psychological challenges, a concept that will be examined in more detail below. While on the surface this problematic contradiction between mental toughness and mental illness appears logical, research has not explored the perspectives of athletes' who have experience with mental illness while taking it into consideration.

As a result of grappling with multiple stressors inherent to the competitive sport context, and while keeping in mind that sport participation can also represent an important coping mechanism for athletes struggling with mental illness by acting as a source of enjoyment and escape from negative events (Jones et al., 2013), researchers in the field of sport psychiatry have pointed out that the relationship between sport and mental illness in athletes is complex. This complexity lies in the very fact that sport may have nothing to do with an athlete's psychological challenges, may be used as a coping mechanism, or may be a primary cause of psychological challenges (Reardon \& Factor, 2010). Due to the possibility that the sport context may play an integral role in an athlete's experience with mental illness, Glick and Horsfall (2009) have advocated for the tailored psychiatric and/or psychological treatment of athletes; treatment that must be adapted to the athletic context. When one considers the evidence that has been outlined above regarding the plethora of stressors that the sport context apparently imposes on competitive athletes, it is reasonable to assume that a counselor or therapist would be more effective at working with athletes with mental health concerns if he or she has a background in or 
specific knowledge and experience with sport. This concept has fueled a small body of research which has examined elite and collegiate-level athletes' perceptions of the characteristics of effective sport psychology consultants and mental health professionals, and in particular the level of importance they place on the practitioner having knowledge of the sport context.

\section{Athletes' Perspectives on Effective Psychological Practitioners}

From a general perspective on providing effective mental healthcare, the Mental Health Commission of Canada published a comprehensive health strategy in 2012 which stated that Canadians were in need of better access to "intensive, acute, and highly specialized services, treatments and supports when they are needed.” (Peachey, Hicks, \& Adams, 2013, p. 4). This concept of highly specialized services can conceivably be applied to the suggestion outlined above regarding the potential importance of a healthcare provider having specific knowledge of sport when working with athletes. However, a suggestion of this nature should be informed by the perspective of the actual recipients of such services.

Research with clinical populations in general has established that rapport and relatedness within the client-therapist relationship is critical to treatment success (Joe, Stimpson, Dansereau, \& Rowan-Szal, 2001; Leach, 2005). Joe and colleagues (2001) examined the relationship between counseling rapport and drug abuse treatment outcomes with over 500 patients and found that a lower level of rapport during treatment predicted worse post-treatment outcomes, mainly in terms of cocaine use and criminality. Alternatively, higher ratings of client-therapist rapport were associated with more positive outcomes on these measures. In addition, Mackenzie and Tscheschke (1993) found that relatedness predicted significantly better outcomes in in-patient clients who were part of a group therapy session. Joe and colleagues (2001) describe rapport between patient and counselor as reflecting "the extent to which the two are on the same 
wavelength and care for one another's well-being” (p. 1224). Similarly, relatedness is a feeling of attachment and comfort with another person or group (MacKenzie \& Tschuschke, 1993).

From a more general perspective, Lambert and Barley (2001) summarized review and meta-analytic research examining the role of the therapeutic relationship in psychotherapy outcomes in an attempt to evaluate the relative impact of extra-therapeutic factors, client expectancy, specific therapy techniques, and common factors (i.e. empathy, warmth, and congruence). Of particular interest to the current inquiry, Lambert and Barley (2001) concluded that the common factors, which capture the nature of the relationship between client and therapist, account for approximately $30 \%$ of improvement in treatment outcomes. This conclusion is consistent with a long history of therapist characteristics being associated with positive treatment outcomes. These characteristics include credibility, empathetic understanding, affirmation of the patient, ability to engage the patient, and focusing on patient's problems (Orlinsky, Grave, \& Parks, 1994). Notably, Lambert and Barley (2001) also observed that when client-perceived relationship factors are assessed as opposed to rater's evaluations of the therapeutic relationship, more positive treatment outcomes are found, thus highlighting the value in exploring the athletes' perspectives of mental healthcare and desirable therapist characteristics.

Many of the concepts and characteristics relevant to the client-therapist relationship that have been discussed thus far can be consolidated by the concept of therapeutic alliance. Therapeutic alliance has been defined by Bordin (1979) as made up of "three features: an agreement on goals, an assignment of tasks or a series of tasks, and the development of bonds" (p. 253). Notably, in their 2003 review of therapist characteristics influencing therapeutic alliance, Ackerman and Hilsenroth (2003) conclude that "the therapist's ability to understand and 
relate to the patient's experience may be an important component in building a strong alliance" (p. 4). Therefore, when considering mental healthcare for a distinct population that faces unique stressors, such as athletes, specific knowledge of or experience within the sport context may be a significant attribute. Our understanding of its role in establishing a therapeutic alliance has yet to be explored in depth. Some research has, however, explored athletes' general perspectives on effective sport and clinical psychology practitioners. In the distinct but related field of sport psychology, researchers have interviewed and surveyed athletes about their perspectives on the characteristics that make an effective sport psychology consultant for helping with performance related concerns (Anderson, Miles, Robinson, \& Mahoney, 2004; Lubker, Visek, Geer, \& Warson, 2008; Weigand, Richardson, \& Weinberg, 1999). Minimal research, however, has investigated athletes' perceptions regarding mental health professionals in the context of treatment for mental illness (Broughton \& Neyer, 2001; Gulliver et al., 2012; Lopez \& Levy, 2013; Watson, 2005).

In terms of the characteristics of effective sport psychology consultants, anecdotal reports from consultants suggest that commitment, passion, strong interpersonal communication skills, effective intervention skills, patience, flexibility, empathy, attentive listening, and a genuine sense of caring are important attributes (Yukelson, 2001). Similarly, Weigand and colleagues (1999) interviewed coaches and athletes of a women's college basketball team who reported that the effectiveness of a sport psychology intern working with the team on mental performance was based on being helpful, knowledgeable about basketball and mental skills, caring, understanding, available, and trustworthy. Anderson and colleagues (2004) were interested in extending this work to elite athletes and interviewed 30 United Kingdom athletes competing at the national level or higher who were either currently working with a sport psychology consultant or had in 
the past. These researchers identified the higher-order themes of personable, good communicator, provider of a good practical service, and knowledgeable and experienced in sport and sport psychology as contributing to being an effective consultant. Regarding the third theme, being knowledgeable and experienced in sport, the athletes suggested that this characteristic contributed to them feeling understood by the consultant and that it was important that this knowledge be sport specific.

Interestingly, Anderson and colleagues (2004) also reported that some of the athletes in their study felt that it could be advantageous for a consultant to not have sport-specific experience because it could allow for a fresh and objective perspective. These contradictory perspectives led the researchers to suggest that "demonstrating an interest in and willingness to learn about the sport is more important than in-depth technical knowledge" (Anderson et al., 2004, p. 266). Lubker and colleagues (2008) surveyed 204 collegiate athletes and sport psychology consultants and found that the most important characteristic of effective sport psychology consultants was positive interpersonal skills, followed by professional status and then athletic background. This finding, of having previous experience with sport as the third most important factor identified by athletes and consultants, led the authors to suggest that sport psychology consultants should disclose any previous experience with sport to their clients, but that this experience is not critical to the consultant's effectiveness. This conclusion is relatively consistent with Anderson and colleagues' suggestions; however, an important distinction exists between the participants in each study. While Anderson and colleagues (2004) interviewed athletes who were either currently working with or had previously worked with a sport psychology consultant, only 67\% of the athletes in Lubker and colleagues' (2008) study had worked with a sport psychology consultant and thus were not necessarily responding based on 
personal experience. Therefore, we can conclude from an analysis of these studies that although a consultant having sport-specific knowledge is considered important to athletes working with such professionals, there is insufficient evidence in the limited existing literature to suggest that it is critical for establishing rapport and relatedness.

Similar findings to the studies outlined above have been reported when athletes are asked to identify characteristics of an effective mental health counselor. These studies, however, involve asking athletes about hypothetical expectations as the majority of them do not include a sample of athletes who have actually used mental healthcare services. For example, Watson (2005) compared the attitudes of 135 collegiate student-athletes with 132 non-athlete students regarding expectations about professional mental health services and found that the athletes had less positive attitudes toward help-seeking behaviour and expected counselors to be knowledgeable and competent. This finding led the authors to conclude that professionals in counseling services should be familiar with the specific demands placed on student-athletes and that "the importance of having environment- or culture-specific knowledge and skills cannot be understated" (p. 447). These athletes, however, were not specifically asked if a counselor's knowledge of the sport context would be a contributing factor to counselor effectiveness.

Lopez and Levy (2013) also surveyed university student-athletes about their preferences for seeking counseling. One hundred and sixty-five athletes from twenty different sports were asked to imagine that they were considering seeking help for a personal issue from professional mental health services and to complete a Counseling and Psychotherapy Preferences Questionnaire which the researchers had adapted from Smith (2005). These athletes indicated that they would prefer to seek help from counselors who had knowledge and experience with sport participation, especially collegiate sport participation. Lopez and Levy (2013) concluded 
that athletes probably prefer to be free to focus on a specific issue troubling them during a counseling session rather than having to explain the intricacies of their sport. While the conclusions and suggestions derived from the results of these studies are logical and potentially valid, without hearing from athletes who have actually worked with professional mental health counselors or therapists, a critical perspective is missing. It may be that athletes who have worked with a mental health professional would have similar sentiments to those athletes in Anderson and colleagues (2004) study who felt that a sport psychology consultant who did not have specific knowledge of their sport offered a fresh and objective perspective. Whether this perspective could apply to counseling that is not focused primarily on performance enhancement, as sport psychology is, but rather is focused on tackling psychological challenges or even mental disorders in athletes, is unknown and has not been specifically examined, particularly with athlete populations other than student-athletes. Indeed, Lubker and colleagues (2008) suggested that athletes at different levels may perceive different counselor characteristics to be important. Therefore, the degree to which athletes of varying levels of sport participation who have undergone psychiatric or psychological treatment feel that a clinician's sport specific knowledge is an important factor in the treatment experience should be addressed.

\section{Access and Barriers to Mental Healthcare for Athletes}

There are also a number of questions surrounding how athletes access mental health care, how they may access a clinician with a specific knowledge of the sport context, and whether this is indeed an important factor for their perceptions of effective treatment. Athletes' access to mental healthcare is an important consideration given the bulk of evidence that points to the existence of numerous barriers to access that may be specific to this population (Gulliver et al., 2012; Kaier, Cromer, Johnson, Strunk, \& Davis, 2015; Lopez \& Levy, 2013; Watson, 2005). In 
addition, research has indicated that athletes tend to underutilize mental health services (Maniar, Curry, Sommers-Flanagan, \& Walsh, 2001; Martin et al., 2001). This finding indicates that some of the barriers that will be outlined below exert a direct effect on whether or not athletes choose to seek out help for psychological challenges or mental illness, and many of them are highly intertwined with the social and cultural context of competitive sport discussed above.

Gulliver and colleagues (2012) reported that the elite athletes with whom they conducted focus groups identified perceived public and self-stigmatizing attitudes toward mental illness in athletes as the greatest barrier to help-seeking behaviors for mental healthcare. Athletes frequently express concern over not wanting to appear weak or as if they are not coping effectively to coaches, teammates, and the general public (Gulliver et al., 2012; Lopez \& Levy, 2013). In addition, these athletes identified lack of knowledge about mental health services, feeling uncomfortable approaching someone for help, time constraints, money, and concern that the healthcare provider would not understand their problems as additional barriers. The concern over a healthcare provider not understanding athletes' problems is highly relevant to the question of whether sport-specific knowledge would enhance treatment effectiveness. Therefore, this particular barrier could be resolved by increased knowledge about the importance that athletes who have undergone such treatment place on this characteristic.

Lopez and Levy (2013) surveyed 165 collegiate athletes about their perceived barriers to mental healthcare help-seeking and identified lack of time to seek services, fear of stigma, and being considered weak for seeking services as significant factors. These researchers noted that the athletes were apparently greatly concerned with the perceptions of others thus making the risk of being recognized at counseling or other mental health treatment centers a strong deterrent from seeking help. This suggestion is consistent with reports by mental healthcare providers who 
have treated high-profile, professional athletes that the concern over being recognized often prevents these athletes from seeking help from accessible sources, such as campus counselling centers in the case of student-athletes (Glick, Stillman, Reardon, \& Ritvo, 2012). Worth noting is that, similar to most of the athlete participants in the counselor effectiveness research outlined above, the athletes in the Gulliver and colleagues (2012) and Lopez and Levy (2013) studies were not sampled based on their previous experience with mental healthcare, therefore their perceptions can be considered speculative.

Two studies have compared attitudes toward help-seeking behaviour for mental illness and stigma surrounding receiving such care in student-athletes and their non-athlete peers. These studies demonstrate the significance of stigma as a barrier for athlete populations (Kaier et al., 2015; Watson, 2005). Kaier compared perceptions of mental illness stigma in 304 university athletes and 103 non-athlete students and found that athletes had significantly higher levels of personal and perceived public stigma. Similarly, Watson (2005) compared the attitudes of 132 collegiate athletes and 132 non-athlete students and found that the university athletes had less positive attitudes toward help-seeking behaviour for professional psychological help. These studies indicate that although stigma is a powerful personal and societal barrier for anyone who may be in need of mental healthcare services (Pedersen \& Paves, 2014), it may be a particularly powerful factor for athlete populations.

Therefore, it is clear that athletes perceive a number of barriers to mental healthcare helpseeking behaviors, and the nature of those barriers has been consistently identified in the relevant literature. What are less clear are the avenues by which athletes who have sought treatment tend to do so. Identifying the avenues by which athletes have been successfully referred to a psychiatrist or clinical psychologist for mental illness treatment may be highly valuable 
information to inform athletes about effective methods and reduce perceived barriers. A study by Mann, Granna, Indellcato, O'Neill, and George (2007) highlights how little is known about how athletes access mental health care. In their survey of 827 sports medicine physicians, three quarters of the physicians reported that they rarely or never referred athletes to sport psychologists for injury-related issues, and two thirds reported that they rarely or never referred athletes for non-injury-related issues. The physicians were not asked about referral to a psychiatrist or clinical psychologist for mental health concerns. Finally, the physicians perceived sport psychologists and athletic trainers or physical therapists to be moderately effective in working with athletes with psychological problems. It is unclear whether these professionals tend to suggest that athletes should seek mental health care. These researchers contend that sports medicine and team physicians are often the first healthcare providers to become aware of emotional and behavioural problems in athletes. In their recommendations for developing a plan to recognize and refer student-athletes with psychological concerns, Neal and colleagues (2015) indicate that team physicians, athletic trainers, and community-based mental health care professionals should all be part of a team-approach to ensure the appropriate referral for studentathletes for psychological care. While this is likely a helpful suggestion, athletes who have successfully accessed psychological treatment have not been asked who referred them or who in their lives became aware that there may be a mental health concern and suggested they seek treatment, if anyone. Exploring these processes may lead to an understanding of which individuals are in fact more influential in the referral process than those identified in the existing literature. Therefore, addressing these questions by asking athletes who encouraged them to seek treatment and who they received a formal referral from will help generate preliminary 
knowledge regarding how athletes may successfully access psychiatric and clinical psychological care.

\section{Conclusion}

The perspectives of athletes on sport and mental health, and accessing mental healthcare, who have direct experience in these areas have received minimal research attention. In addition, the exploration and interpretation of these perspectives while being cognizant of prevailing discourses in competitive sport surrounding mental health, mental toughness, weakness, and vulnerability is critical to understanding the various social and cultural influences on the athletes' experience. Specifically, the importance that athletes place on their psychiatrist or clinical psychologist having a specific knowledge of sport for successful mental illness treatment is an understudied topic. This question should be asked of athletes who are currently receiving treatment from a mental healthcare provider or have a history of doing so. This inclusion criterion would address the gap in the current research that has developed as a result of simply asking athletes to speculate on therapist characteristics that they would deem important rather than recruiting participants who are in a position to reflect on first-hand experience. In addition, the experiences of athletes from multiple levels of competitive sport participation should be explored - including university, national, international, and professional - to determine whether preferences regarding a therapist's specific knowledge of sport is impacted by the level of competitive involvement. The inclusion of athletes from multiple levels of sport will also expand the existing literature on the topic which has relied predominantly on university athlete samples. Furthermore, investigating the avenues by which athletes who have received mental health treatment accessed such treatment and who suggested they do so may provide valuable 
information about how athletes have successfully navigated the mental health system and overcome access barriers.

\section{Chapter 2: Methodology and Methods}

The majority of research investigating athletes' perceptions of their experiences with sport psychology and mental health services has utilized quantitative measures, such as selfreport surveys (Lubker et al., 2008; Watson, 2005). However, the interview-based work of Weigand and colleagues (1999) and Anderson and colleagues (2004) demonstrates the depth in understanding that can be achieved by utilizing such methods. Although survey-based studies are useful in the assessment of prevalence, general perceptions, specific predictors, and possible causal pathways these designs do not allow insight into the individual experiences of athletes who describe mental health challenges and mental healthcare interactions. The point of view of the athletes, unconstrained by surveys, may illuminate some of the unanswered questions in the current literature and provide valuable knowledge about treating this unique population. Qualitative research is, in fact, an umbrella term for a range of specific methodologies and methods, and each of these is informed by attendant epistemological underpinnings (Chamberlain, 2000). The current inquiry will build on existing qualitative work in this area by adopting a social constructionist perspective to highlight the value of interpreting the athlete perspective as a meaningful contribution to our understanding of mental health treatment, and through the lens of the broader social and cultural influences inherent in competitive sport.

\section{Research Questions}

1. What is the role of sport culture in the psychological stressors that athletes with mental health challenges face? 
2. What importance do athletes place on their mental healthcare provider having a specific understanding of the sport context for effective treatment?

3. What are the avenues through which athletes tend to access mental healthcare?

\section{Paradigmatic Perspective: Social Constructionism}

The current investigation was informed by social constructionism, an epistemological approach which takes a critical stance toward "taken-for-granted" knowledge and encourages awareness of the context in which that knowledge is produced (Burr, 2015, p. 2). By exploring athletes' perspectives of their subjective experiences with mental healthcare, this project took an approach to evaluating psychological treatment that is in contrast to mainstream treatment efficacy research. Social constructionism challenges the traditional, often realist and reductionist, approaches to investigating psychological phenomena by arguing that knowledge and understanding are specific to the historical and cultural context in which they are produced. Rather than searching for a universal and generalizable answer to my research questions, which would reveal an assumption that mental healthcare experiences can be distilled to specific fundamental truths shared by all athletes, I sought to learn about how athletes construct their perspectives on mental health and healthcare given the discursive resources available to them in sport culture.

According to social constructionism, the assumption is not only that peoples' realities are specific to the broader historical and cultural processes in which they are situated, but that these

processes also produce understanding (Burr, 2015). For example, I approached the current inquiry with the assumption that the athletes' perspectives on sport, mental health, and mental healthcare have been produced not merely by internal and individual processes, but by the expectations, assumptions, and accepted norms for behaviour within competitive sport culture 
that were outlined in the above literature review. Within a social constructionism paradigm, a range of ontological positions may be applied (Burr, 2015), however in the current study a relativist ontological stance was taken. This stance holds that each athlete's perspective, and the way in which he or she shares that perspective within the social context of a research interview, reflects an alternative but equally valid conceptualization of reality (Guba \& Lincoln, 1994).

As I have already alluded to, a fundamental assumption of social constructionism is that knowledge is actively produced through social processes and language use (Burr, 2015).

Therefore, the use of qualitative, interview-based data is appropriate within this paradigm. This assumption is also applied in the current inquiry by the understanding that my and the athletes' knowledge about the topics discussed was produced through the social interactions we have formerly had, and through the conversation that unfolded during the interviews. These ideas are related to questions of epistemology, which is the study of knowledge production, including the relationship between reality, or that which is being studied, and the researcher. Epistemology also refers to questions of what types of knowledge are considered legitimate and what forces, such as institutional, socio-cultural and discursive, shape knowledge production and dissemination. From the social constructionist perspective of the current inquiry the role of the researcher's decisions and past experiences, based on his or her personal and sociopolitical understandings, in the knowledge produced is acknowledged (Burr, 2015; Creswell, 2007). In addition, the athletes' individual perspectives are considered valid accounts that represent their reality and understanding of their experiences. Explicit acknowledgement of this role of the researcher and multiple realities based on individuals' particular context and experiences differs from the objectivist perspective that is frequently applied in positivist clinical psychology research. Such approaches typically assume that the researcher should be as removed as possible 
from the scientific data to limit bias and that one reality exists to be revealed through scientific inquiry (Daly, 2007); in contrast, social constructionism acknowledges that research is unavoidably subjective. Subjectivism also implies that knowledge production is transactional, a joint-production between the researcher and participant (Guba, 1996). Therefore, the researcher is not an unbiased observer and recorder of the participants' behaviours and experiences, rather he or she brings knowledge and perspective into the interpretive process of qualitative inquiry.

\section{Method}

\section{Participant Inclusion Criteria}

Ten athletes participated in the study. Inclusion criteria encompassed male and female identified, aged 18 and older, current or former competitive athletes who have experience with psychiatric or psychological treatment for a mental health concern. Participants may be currently undergoing treatment or have had experience with treatment in the past. A variety of levels of competitive athletes were recruited to explore whether competitive level plays a role in athletes' experiences with mental health treatment. Therefore, interuniversity, elite, and professional athletes were recruited. The extent of mental healthcare treatment that individuals had undergone was such that they had the opportunity to establish an understanding of the clinician's specific knowledge of the sport context.

\section{Data Collection}

Data was collected in two phases. Interviews had already been conducted with five athletes as part of a pilot phase of the inquiry to develop a sense of the experiences that athletes have with mental health and healthcare. These data informed the revision of the interview guide to incorporate questions more specifically designed to inquire about athletes' perspectives within the social and cultural context of competitive sport (See Appendix A for Interview Guide). 
Sample interview questions include: "How would you describe competitive sport culture?"; "How do you think athletes perceive mental illness?"; and "What do you think is important within the therapist-patient relationship for effective mental health treatment?". The second phase of the study, using the revised interview guide, focused on recruiting additional competitive athletes. The following outline of data collection methods is very similar to the procedure used in the pilot phase; however, it pertains specifically to the second phase of data collection.

Participant recruitment. Strategies for recruiting athletes for the pilot phase of the study were successful and therefore were implemented in the second round of data collection. Two specific strategies were used: recruitment posters were placed in athletic facilities on the University of Toronto and Ryerson University campuses, attracting both interuniversity and community athletes (see Appendix D for Recruitment Poster); snowball sampling was also used, a technique that involves accessing initial participants and asking them connect the researcher to potentially interested participants who fit the study inclusion criteria (Morgan, 2008). This strategy was facilitated by my participation in the sport of track and field. I reached out to athletes who had discussed their experiences with mental health issues with me in the past or publicly via social media. After connecting with a few interested participants, I asked the athletes to reach out to other individuals who fit the inclusion criteria of the study. These athletes were asked to provide my email address to interested individuals, ensuring that participation was their choice. Because of the sensitive and potentially distressing nature of discussing mental health issues, before meeting with each interested athlete, I provided them with an electronic information and consent form so athletes could gain more familiarity with the details of the study 
and have the opportunity to decline participation at that point. If the athlete was still interested, we scheduled an interview via email.

Interviews. Individual, semi-structured interviews were conducted with each participant. The interviews were 45 to 75 minutes in length and took place in a private interview room at the Ryerson University psychology labs at a time and date that was mutually convenient for both researcher and participant. The nature of the semi-structured interview is consistent with the social constructionist paradigm which informs this inquiry. While it represents a guide to inform the direction of the conversation based on the research questions of interest and provides a degree of topic consistency across individuals, it also allows participants to elaborate on particular topics or questions that are most salient to them. This approach to the interviews is also consistent with Fontana and Frey's (2000) emphasis on the importance of flexibility within qualitative interviewing in order to reduce the limitations of imposing a priori categorization to the field of inquiry. Engaging in an interactive style of interviewing (Ellis, Kiesinger, TillmannHealey, 1997) also emphasizes the intent inherent in constructionism of both researcher and participant contributing to joint sense-making of the conversations that unfold (Burr, 2015). The interviews began with demographic and descriptive questions (e.g. age, type and level of sport participation). The mental health component of the interview focused on the following domains: general perceptions about mental health and competitive sport culture; personal experiences with sport, mental health, and mental health treatment.

Reflexive journal. Inherent to social constructionism is the assumption that meaning is created through social interaction, thus during interviews both research participants and the researcher play a role in the co-construction of conversation and its meaning (Burr, 2015). Therefore, engaging in reflexivity throughout the research process was critical to identifying and 
interpreting the values, experiences, assumptions, and personal social identities that $\mathrm{I}$, as the researcher, am influenced by and which shape my perspective (Willig, 2004). Reflexive awareness was an ongoing exercise throughout the design, implementation, and analysis phases of a given inquiry, and was facilitated by keeping a reflexive journal. Therefore, I made regular entries in a journal, documenting my unfolding thoughts and ideas and their influence in my interpretations and analytical decisions. The journal served as a tool to be critically aware of my position within the inquiry, as well as a resource for ideas during data analysis.

\section{Data analysis}

Braun and Clarke (2006) advocate for the utility of thematic analysis in qualitative research as it is a flexible approach that allows for the opportunity to provide a rich and complex account of the data and is malleable to the particular epistemology within which it is used. Thematic analysis is also appropriate for conducting largely exploratory and inductive inquiries, such as this one, because of its flexible implementation (Vaismoradi, Turunen, \& Bondas, 2013).

Specifically, thematic analysis involves the researcher creating themes based on identified common ideas and concepts across a collection of interviews (DeSantis \& Noel Ugarriza, 2000). A theme is defined by Sandelowski and Leeman (2012) as a "coherent integration of the disparate pieces of data that constitute the findings" p. 1407. Braun and Clarke (2006) further describe a theme as capturing something meaningful within the dataset that is typically found across multiple participants' accounts, although this is not to say that the number

of accounts that hold evidence of a theme necessarily speaks to the theme's importance. Rather, the extent to which a theme describes something that is interpreted as meaningful by the researcher in relation to the research questions is a more appropriate measure of its relevance. 
No singular and prescribed process is advised; rather researchers may draw on general guidelines to structure their analytic process. For example, Braun and Clarke (2006) outline a number of steps that were used to guide analysis in the current inquiry. The first phase of data analysis involved familiarizing myself with the interview transcripts by conducting multiple readings. These readings initiated the process of meaning-making by paying attention to commonalities as well as points of divergence among the athletes' accounts. Second, initial codes and data extracts were noted in the margins of the transcripts that highlighted potential meaningful concepts. Initial codes stayed "close" to the data, in the sense that they reflected what was stated by the athletes as opposed to incorporating higher order interpretation, which occurred in the next stage. Following this preliminary coding stage, I divided and combined the initial codes into potential higher-order and sub-themes. At this step of the thematic analysis interpretation began to play a bigger role in informing how the initial codes and data extracts were most meaningfully represented by themes. Next, I engaged in a process of reviewing and revising the themes to solidify their value in capturing the content and nuance of the data, before specifically defining and naming each retained theme.

Consistent with a social constructionist perspective, particular attention will be paid to what Braun and Clarke (2006) describe as latent or interpretative themes. This means exploring broader meanings and implications that are believed to influence the semantic content of the interview data, such as the role of the sociocultural context of competitive sport in athletes' experiences with mental health. In this way analysis moves beyond a purely descriptive level to incorporate interpretation that exposes underlying assumptions about knowledge production and our understanding of human experience. 


\section{Chapter 3: Findings}

The thematic analysis of the athletes' experiences and perspectives on mental health and mental healthcare in the sport context was broadly structured by three research questions: 1) What is the role of competitive sport culture in the psychological stressors that athletes face and how they perceive mental illness?; 2) What are athletes' perspectives on the importance of their mental healthcare provider having a specific understanding of the sport context?; and 3) What are the avenues by which athletes access mental healthcare, including the barriers and facilitators to accessing care?. The transcribed interviews were first coded based on data that were relevant to these questions and subsequently organized into higher-order themes and sub-themes described below.

The perspectives that the athletes shared are assumed to be informed by their individual sport contexts, as well as the social and cultural dynamics influencing their lives more broadly. Therefore, a profile of participant characteristics is included in Table 1 for the reader to gain an understanding of the context in which each participants' perspective is embedded. Although

there was a range of specific competitive levels between the athletes, for the sake of consistency throughout the document they will be described as "competitive athletes" based on Van Slingerland and colleagues' (2019) definition adapted from Araújo and Scharhag (2016). Consistent with this definition all athletes devoted several hours to training and competing in their sport that exceeded time devoted to other extracurricular activities, and all regularly participated in competitions at the provincial, national, or international level. Other language used throughout the document requiring definition are the terms mental illness, mental disorders and mental health challenges. Mental illness is used interchangeably with mental disorder to describe clinically significant alterations in individuals' feelings, thinking and behaviour 
associated with significant distress and impaired functioning in important domains of life (Public Health Agency of Canada, 2006). This terminology is used when analysis is based on experiences where the athletes have identified a specific mental disorder diagnosis. Mental health challenges is used to describe psychological distress more broadly and when clinically diagnosable symptoms may not necessarily be present, but mental health is still being negatively impacted.

Table 1.

Participant Characteristics

\begin{tabular}{|c|c|c|c|c|c|}
\hline $\begin{array}{l}\text { Athlete } \\
\text { Pseudonym }\end{array}$ & $\begin{array}{l}\text { Age } \\
\text { (years) }\end{array}$ & Race & $\begin{array}{l}\text { Sport(s) } \\
\text { (current/retired) }\end{array}$ & $\begin{array}{l}\text { Years of } \\
\text { sport } \\
\text { participation } \\
\text { (years) }\end{array}$ & $\begin{array}{l}\text { Competitive } \\
\text { level* }\end{array}$ \\
\hline Brenda & 39 & White & $\begin{array}{l}\text { Figure skating } \\
\text { (retired) and Track } \\
\text { and Field (current) }\end{array}$ & $\begin{array}{l}\text { Figure } \\
\text { skating (11) } \\
\text { Track and } \\
\text { Field (14) }\end{array}$ & $\begin{array}{l}\text { Figure skating } \\
\text { (National) } \\
\text { Track and } \\
\text { Field } \\
\text { (International } \\
\text { Masters } \\
\text { Division) }\end{array}$ \\
\hline Heather & 18 & White & $\begin{array}{l}\text { Volleyball } \\
\text { (current) }\end{array}$ & 5 & $\begin{array}{l}\text { National and } \\
\text { interuniversity } \\
\text { sport }\end{array}$ \\
\hline Justin & 23 & White & $\begin{array}{l}\text { Hockey (retired) } \\
\text { and Volleyball } \\
\text { (current) }\end{array}$ & $\begin{array}{l}\text { Hockey (5) } \\
\text { Volleyball } \\
(4)\end{array}$ & $\begin{array}{l}\text { National and } \\
\text { interuniversity } \\
\text { sport }\end{array}$ \\
\hline Addison & 35 & White & $\begin{array}{l}\text { Track and Field } \\
\text { (current) }\end{array}$ & 22 & International \\
\hline Jasmine & 29 & White & $\begin{array}{l}\text { Track and Field } \\
\text { (current) }\end{array}$ & 17 & National \\
\hline Clara & 27 & White & $\begin{array}{l}\text { Track and Field } \\
\text { (current) }\end{array}$ & 10 & International \\
\hline Mia & 24 & White & $\begin{array}{l}\text { Track and Field } \\
\text { (current) }\end{array}$ & 7 & $\begin{array}{l}\text { National level } \\
\text { and } \\
\text { interuniversity } \\
\text { sport }\end{array}$ \\
\hline Emma & 24 & White & $\begin{array}{l}\text { Swimming } \\
\text { (retired) }\end{array}$ & 6 & $\begin{array}{l}\text { Junior national } \\
\text { and } \\
\text { interuniversity } \\
\text { sport }\end{array}$ \\
\hline
\end{tabular}




\begin{tabular}{|llllll|}
\hline Raevyn & 24 & Black & $\begin{array}{l}\text { Track and Field } \\
\text { (current) }\end{array}$ & 9 & International \\
\hline Pierre & 24 & White & Diving (retired) & 12 & International \\
\hline
\end{tabular}

*Competitive level definitions: Interuniversity sport - participated in national university sport competition; National - competed at the highest national level and in international competitions without representing his/her country; Junior national - competed in the junior age category (under 19) at the highest national level; International - competed internationally while representing his/her country.

\section{What is the Role of Competitive Sport Culture in the Psychological Stressors that Athletes Face and How They Perceive Mental Illness?}

The athletes were asked to describe competitive sport culture and the relationship between their mental health and their experience as an athlete within that culture. Through exploration of these topics it was clear that all the athletes perceived both positive and negative aspects of competitive sport culture to play a role in the manifestation and course of their mental health challenges, and in their perceptions of mental illness. The data that illustrates this complex relationship is presented using three higher-order themes. The first theme, "The Normative Discourse of What it Means to be a Competitive Athlete", describes what being a competitive athlete entails based on both the athletes' internalized expectations and perceived others' expectations. The second theme, "The Experience of Being an Athlete with Mental Illness", provides a detailed portrayal of the positive and negative elements of sport that the athletes perceived were related to their mental health. Finally, the third higher-order theme that addresses this research question is, "Athletes Understandings of Mental Illness Within the Context of Sport", which describes how this group of athletes perceives mental illness in light of the normative discourse of being a competitive athlete described in the first theme. The sub-themes described in "Athletes Understandings of Mental Illness Within the Context of Sport”, illustrate attitudes that are the consequence of athletes' and others' perceptions of a normative competitive athlete being misaligned with the reality of the experience of an athlete with mental illness. 
The Normative Discourse of What it Means to be a Competitive Athlete. In reflecting on their experiences as competitive athletes and their relationships to their sports, the athletes painted a picture of the internal and perceived others' expectations of what being a competitive athlete means. We can understand the nature of these expectations by exploring the role that the athletes described embodying during their pursuit of athletic success.

Athletes' perspectives on the competitive athlete role. The competitive athlete role is characterized by complete commitment and dedication to the athletic pursuit and is produced by the dominant culture in competitive sport that many of the athletes described as prioritizing performance over athlete wellbeing. A representative example of this perspective is seen in Pierre's description of the competitive culture in his sport:

Pierre: In competitive sport the culture is to focus on performance and outcome. We value results and medals over the wellbeing of the athletes. The way we monitor success in sport is through medals, not whether you're growing as a person, whether you're happy, because at the end of the day if you're not performing well the sense is why does it matter if you're a happy person? I find that really upsetting.

Many of the athletes described the nature of their sport participation as involving an extreme level of commitment and dedication to achieve the outcomes valued by this culture. According to Pierre, these outcomes are achievement-based, which creates an emphasis on the ends versus the means and subsequent neglect of athlete mental health. Various sources of pressure produced by the culture of competitive sport described above, including internal and external expectations, normalized a level of rigid commitment that led to the deterioration of other domains of the athletes' lives such as academics, physical health, and relationships. Clara 
described the pressure she feels in her sport to prioritize complete commitment to it and thus sacrifice other pursuits:

Clara: It's a 24 hour job in the sense that I'm aware of what my competitors are doing off the track, outside of training, so I'm aware of that 24 hours a day as well. I had so much guilt associated with missing a training session. There is a feeling that you have to do it because this is what you've chosen your job as. I tried to be successful in running, I tried to be successful in my relationship, and I tried to be successful in my job. It took me three years to realize I can do one of those really well and maybe a second moderately well, but the three of them together was a recipe for disaster. I just had to sacrifice constantly for the other ones.

Brenda also described the commitment to performance success at almost any cost that was expected within the culture of competitive figure skating as leading to sacrifice, in this case her physical health as she was encouraged to ignore injuries in order to perform:

Brenda: Toward the end it involved skating two times a day, missing school, attending summer practices that were seven hours a day. The year I quit skating I was finally able to see it from a more objective point of view and realize how crazy it was, how unhealthy it was. I was very injured that summer. I had torn a hamstring and they wouldn't let me rest. I remember literally icing my hamstring until I couldn't feel it, bandaging it up, having numbness down my leg, it was awful. I also had a bit of a fracture in my tailbone from falls on triples and what not and I was forced to still do them.

Justin's reflections on his experience echoes the underlying message that Clara and Brenda received within competitive sport culture; that to achieve optimal performance there must be an unquestioning commitment to and prioritization of sport, and that relationships and employment 
opportunities must be sacrificed if they interfered with this commitment. Justin described this normative attitude in youth hockey culture:

Justin: Athletes are taught that you have to really invest to get anything out of the sport. If you want to go pro it has to be the only thing you care about. We're definitely fed that idea, that if you're planning for something else you are not fully committed to what you are doing now.

Related to the concept of complete commitment and dedication, Clara described the role of a competitive athlete as generalizing to an all-encompassing lifestyle, such that the rest of her life became structured to facilitate training and competing:

Clara: Overall my experience has been very lifestyle changing, and really all inclusive. I don't want to say everything comes secondary to running...but my life seems to be planned around training now. A lifestyle too in the sense of the people I'm surrounding myself with. If I'm being totally realistic, I lost the tightness of the connection with friends that have nothing to do with the sport as I started to get more seriously into running.

Similarly, Pierre and Emma described the process of committing to and prioritizing sport performance becoming a lifestyle, and they also identified the effect of this process on narrowing athletes' identities and sources of self-worth:

Pierre: A lot of athletes go through the intensity of training, they get good and it becomes a full-time job where you're at the pool every single day. You start pulling back on school, you start sacrificing your social life and you become very narrow minded in what you're doing and what you're pursuing. That process results in essentially your everything becoming your sport. 
Emma: I think with sport athletes become so focused that it structures everything. You go to practice during the day, you have school and you're focused on that, but you have one distraction and its sport and that's what you're thinking about. If you're going to be an elite athlete you have to dedicate yourself to it.

Through exploring these athletes' experiences pursuing competitive sport and reflecting on their lives as athletes, it is clear that they viewed total commitment to sport and the resultant sacrifice of prioritizing other parts of their lives as the normal and expected avenue by which success could be achieved. Evident in these excerpts is also the athletes' critique of the choices they made and felt were imposed by their sport cultures; at the same time as acknowledging the necessity of sacrifice for performance they identified negative repercussions for wellbeing. In the next theme we will see how these athletes' perceptions of others' assumptions further shapes a normative understanding of the characteristics of a competitive athlete.

Perceived others' assumptions about competitive athletes. Some of the athletes discussed what they believe are public assumptions about the characteristics of competitive athletes. Public perceptions were internalized as pressure to fulfill these external expectations and were described as largely inconsistent with the reality of these athletes' experiences.

Emma, Clara, and Brenda identified the public perception that because of their ability to perform in sport at a high level athletes must inherently be high-functioning and possess a mindset impenetrable to the stressors and challenges faced by most individuals. Emma revealed how salient this public perception was to her when she discussed the pressure she felt to live up to this expectation when she found herself being unable to continue pursuing her sport due to injury: 
Emma: I feel like athletes are perceived as being very strong and very put together and indestructible. So you kind of get that mentality and stress when you don't feel that way, so I definitely didn't want people to know I was like that. It actually got worse after I quit and I think part of that is because I lost that identity and thought people probably still perceived me this way and now I'm even less that way because I'm not swimming anymore. It's definitely harder as an athlete when you're perceived in this limelight that you're all put together and really you're just a normal person.

Emma is describing the process by which external expectations of the characteristics of a competitive athlete shaped her athletic identity and the identity loss that occurred when she was unable to maintain her athlete role. Similarly, Clara described her perception that others "assumed that because I was an athlete, captain of my team, involved in the sport community, that I must have my shit together." Like Clara, she felt that her experience did not coincide with this portrayal of a competitive athlete, though there was a desire to uphold others' expectations: "It wasn't that way at all but I was very good at reflecting that image". Finally, Brenda also highlighted the assumptions she perceives the public have about competitive athletes, namely that being apparently high functioning in terms of sport performance means that hardship in other domains does not exist for these individuals or at least is always overcome. There is an incongruence, however, between this view and the reality of athletes experiencing mental health challenges:

Brenda: An Olympic or high level athlete is a perfect example of a person who society views as having a perfect life and has everything. Those people are viewed as people who can't struggle. So I think that perception of anyone that is successful in sport, or anything 
else, that you just don't assume that these people can be succeeding despite their struggles.

The Experience of Being an Athlete With Mental Illness. We shift focus now to examining the relationship between these athletes' mental health and their sport experiences. The athletes discussed a range of stressors and positive elements of pursuing competitive sport that impacted their mental health, and they shared their perspectives on how their mental health impacted their sport performance. By highlighting these perspectives, we gain a nuanced understanding of the role of this environment in the manifestation of mental heath challenges in this population and in athletes' ability to cope with psychological distress. Beyond shedding light on this complex relationship, the analysis presented here serves two additional purposes: to demonstrate how these athletes' experiences at times were misaligned with the normative portrayal of elite athletes described above; and to provide rich context for the second two research questions addressed in this thesis.

Sport and mental health are intertwined. Almost all the athletes described their mental health and sport experiences as being intimately intertwined. Specifically, that competitive sport participation both exposed them to stressors that negatively impacted their mental health and to positive experiences that they perceived as beneficial for their mental health. Before describing the specific stressors the athletes identified, Mia and Pierre's reflections capture the general inter-relatedness of their mental health and sport participation:

Mia: If you're an athlete with depression sport is part of your life and depression is part of your life and it's all mixed together. So if you're going to get help it's going to effect both, no matter what. No matter if you're aiming it at the sport or just the depression or other stuff it all comes together. Depression definitely did affect sport. 
Pierre: I think part of it is having a personality type that puts you at risk for developing something like an eating disorder but then it can be the sport that ignites the flame that causes it to happen. It wasn't until my ninth year in the sport that I started experiencing these thoughts, so I don't know if it takes nine years to slowly chip away at you and the accumulation of small comments ends up making you feel totally inadequate. I don't know what level of resilience it takes to just turn a blind eye to all of that, particularly when you've dug yourself so deep in this hole of committing to your sport.

Interestingly, Pierre highlights both the role of predisposing characteristics that may have drawn him to competitive sport in the first place and the sport context in the development of his psychological symptoms. He also describes a cumulative effect of the demands of competitive sport participation on his mental health, that over time his resilience to the stressors he faced in his sport deteriorated. In addition, both Mia and Pierre suggest that the inter-relatedness between sport participation and mental health is to some extent inevitable given that sport has clearly become linked with their sense of identity and self-worth, a concept that is further explored as one of the specific elements of competitive sport participation that was perceived to impact mental health. To further highlight the connection these athletes described between their mental health and the sport context, Clara's summary highlights other stressors that will be elaborated on:

Clara: It has become something I've invested so much time and energy and finances into and sacrificed for. Obviously with injury, sickness, and other life stressors that come into the picture, it has really played with my mental health and overall happiness. So that's something that I am anticipating will continue to be a challenge, but I think through sport 
I've learned to adapt in different situations and find different tools and resources to help me get through some of those harder times, the challenges.

Expanding on the connection that Mia and Pierre drew between their athletic identities and mental health, Clara and Emma described how their perception of their self-worth was linked to maintaining this identity. Therefore, in the face of obstacles such as injury or lapses in motivation that made it challenging to satisfy this source of self-worth the athletes experienced negative emotions of guilt, frustration, shame, inadequacy, and sadness that negatively impacted their mental health:

Clara: I had so much guilt associated with missing a training session. There is a feeling that you have to do it because this is what you've chosen your job as. There is stigma in the running world about taking a day off or not getting your workout in. Things like that can really affect my mental health. Just like a validity standpoint of my self-worth. Emma: I experienced stress when I got injured and quit because I lost that identity of being strong and indestructible. I didn't feel that way and I definitely didn't want people to know I was like that. It got worse after I quit because now I'm even less that way because I'm not swimming anymore. As soon as I didn't feel like I had it together in terms of sport I felt like I had to have it together in other areas but that just all blew up.

Pierre further commented on how drawing on sport as a primary source of self-worth was related to his mental health, in this case because it led him to evaluate his core value based on performance and to his mood being dependent on performance success. Furthermore, he attributed this negative repercussion of developing a strong athletic identity to the glorification of successful athletes in competitive sport culture: 
Pierre: If I was winning, I was good, if I wasn't winning, I was bad. That's how your mood fluctuates. It was so unhealthy. So much of what I had done, my relationships with people, they were all built on my sport. At my school it was always how I was introduced; it was all I was to everybody. That is what the culture of sport breeds. I walked down that path and it led me to a really dark, upsetting, depressing place. Given this one-dimensional connection between an athletes' self-worth and maintaining the identity of a competitive athlete, we can understand the vulnerability to experiencing significant psychological distress that is produced by performance failure in this context. For example, Pierre attributed the further deterioration of his mental health in the form of worsening depression and disordered eating symptoms to a sense of loss of control and self-worth in the face of sport performance issues:

Pierre: I started feeling like everything I had, which was my athletic performance, was slipping away from me. I started feeling like I was losing control so I resorted to things I could control, which was eating. It turned into an overwhelming depression that I just didn't know how to manage.

Emma, who also experienced disordered eating while she was an athlete, spoke similarly about the role that a decline in her sport performance played in worsening her symptoms as this powerful source of self-worth felt like it was slipping out of her control. For these athletes the loss of control that was experienced in the rupturing of the successful athlete role occurred because they had no other sources of self-identity to draw on, making performance failure feel catastrophic:

Emma: I find that if you're not performing well it's like the end of the world. It feels like it's all you and it's all performance-based. Once the bad performances reached an 
extended period of time, it felt like nothing I did would make a difference. Part of the reason why I think this happened [developing disordered eating] is because of that loss of control. I had gone from always performing really well to not. I think part of the reason I got almost addicted to the sport was because I was doing so well so then when that stopped it was almost a panic, it felt like I had lost control over a lot of aspects of my life. So what I ate and how I looked was definitely something I could control.

From a slightly different perspective, Mia implied the negative impact poor performances in her sport had on her view of her self-worth when she discussed the benefits she derived from psychological treatment:

Mia: Working on positive thinking for everything definitely helped for sport because it helped with everything. It made me feel better in sport because it made me feel more grateful and helped me mature and just not be so negative on myself for bad performances and instead focus on the good things about it.

Another salient stressor associated with competitive sport participation that some athletes identified as significantly impacting their mental health was experiencing injury or illness. This was a stressor because it negatively impacted their ability to achieve athletic success, which has been established as a trigger for psychological distress because of its impact on self-worth and identity. Injury or illness was also perceived as a stressor by some athletes because it interfered with their ability to maintain the structure and normalcy of their day-to-day lives and satisfy the physiological and psychological benefits they felt they derived from training for their sport. For example, in discussing a long-term injury Clara described a level of dependency she has on running to satisfy her identity and emotional needs: 
Clara: I use the analogy of when I was injured it feels like you get into this place where you're essentially not brushing your teeth anymore. Like you're so used to doing it in the morning and evening that when it's taken away from you, I really didn't feel like I was doing my proper daily routine. It can be the best thing for my mental health but when I'm injured and all I want to do is run and I'm seeing everybody out there competing it just feels like there's a piece of me that's totally missing. I get to the point where I feel like the only thing that can cure this sadness and loneliness and feeling of being bloated or not looking how I want to look in my athletic body, is running.

As soon as I was able to start running again after a long injury I just instantly felt myself having more energy in the day, I felt like I started sleeping better, I was in such a better mood when I was around people, I was more invested and conscious of conversations I was having.

Similarly, Emma described how injury interfering with her ability to do her sport meant that she lost a coping mechanism she had become reliant on for keeping her disordered eating symptoms under control: "When I was having injury problems a lot of control was swept away and I relapsed. I ended up having to stop swimming because of my injuries and then the eating disorder got really bad again.”

Brenda also reflected on the distress associated with an illness that prevented her from training for and competing in her sport being due to her identity being so tightly associated with her athlete self:

Brenda: A year and a half ago I was diagnosed with an illness and started undergoing intense treatment. I was told I could never run again. As you can imagine when you're a runner and love it that much that is really hard to deal with. To be forced to become a 
normal girl who goes to spin classes and yoga and not competing in anything when I've been competing since I was six years old?

The final sport-specific stressor that these athletes identified was related to their body image. Pierre and Emma perceived the pressure they felt within their sport cultures to maintain a specific body shape and size to achieve optimal performance and maintain perfectionistic aesthetic standards of an athlete body, as playing a significant role in their development of disordered eating and, in Pierre's case, severe depression symptoms:

Pierre: There are stressors that come with an aesthetic sport. You're in this tiny little speedo and being judged. You're constantly told "this guy is more muscular than you, this guy's stomach looks better, he has a better toe point, he has better hair". There was constant pressure to be thin but muscular, but also to be attractive and have nice hair, all these crazy things. You're bombarded and over time it adds up to where you start developing thoughts that you're not sufficient, your body is unattractive. I developed an eating disorder because I was so calculated in what I was eating and constantly working out. I knew it was irrational behaviour but for some reason I couldn't stop. I knew it was wrong but the desire to be the best outweighs the thoughts that it's not healthy. Eventually it resulted in suicidal thoughts. I was so far gone that it felt like there was no point in trying to dig myself out of the hole, that it would take too much effort so it would be easier to end it all.

Emma: My new coach weighed us every week. In the beginning it didn't affect me that much, I was still performing well and I wasn't too worried about it. But in the end that definitely made it more stressful. When I'm already going through this struggle and then I have to weigh myself every week...it was really bad. 
While Clara and Brenda also discussed body image-related distress, for these athletes it was associated with their bodies changing when they were injured or ill and thus unable to maintain their ideal athletic body shape. For example, Clara said "when I wasn't able to run for so long I felt bloated, not in my normal body or not looking how I wanted, or just feeling how I'm used to feeling in my athletic body." Echoing this perspective, Brenda said, "It was frustrating, losing my jumps, having a different body. That last summer of skating became a complete living hell where for the first time I was looking for excuses not to go."

Important to highlight is that this group of athletes also perceived sport and their mental health to be intertwined because of a range of benefits they derived from their participation. Many athletes viewed participating in sport as a critical coping mechanism for psychological distress they perceived as being associated with factors outside of sport. For example, Brenda and Clara described their perceptions of running as a coping mechanism very similarly:

Brenda: Running improved my mental health and made me feel better about myself. I have dealt with anxiety and depression since I was really young, and running was like a release. I would go for a run and just wouldn't think; my brain would just turn off. With running the negative thoughts would just go away, which I found addictive. Also, just the endorphins from it. I'm certainly one of those people who gets a huge high off a run. Clara: I can definitely relate to the idea of just like having a lot of exterior stress going on in life and using running as a therapeutic time to just get out and get away from the screen, get away from being indoors, get fresh air, get those endorphins, those things have definitely helped me. 
Addison also perceived that running was related to her mental health because of its role in helping her cope with anxiety: "One of my coping strategies was running. I think one of the reasons in the end I didn't get a prescription was because I could go for a run and just think."

Brenda and Heather also identified learning transferable skills as a positive element of the dedication and commitment they brought to training for and competing in their sport. For example, despite experiencing significant stressors in the context of competitive figure skating when she was an adolescent, Brenda emphasized the skills she derived from having to meet the demands of training and competing:

Brenda: Skating instilled discipline at a really young age, and how to really dedicate to something. I don't think without skating and the crazy regimen I had since I was a little kid I would have become a doctor. Just getting through medical school and the studying that was required, the time management, you know. I think it has been what has allowed me to succeed in other areas of life, especially academically. I think that is what it has given me, that ability to really dedicate to something and stick to a plan.

In a more general sense, Heather also described the value she attributes to her sport participation as related to the skills she has learned and the overall benefit it has had for her mental health:

Heather: I love sports. I don't have enough good things to say about getting kids involved in sports. I've learned things from sports, like teamwork and leadership skills. I think it has just helped me have a really good understanding of other people and listening to their ideas. I think we tend to forget how good it is for your mental well-being and your professional well-being.

The importance these athletes placed on the positive elements of their experiences as competitive athletes is reflected in many of them concluding that overall they value rather than 
regret their commitment and dedication to sport. Pierre shared a salient example of this sentiment when he described the personal growth he attributes to both the negative and positive experiences he had as a competitive athlete:

Pierre: It's an experience I would never trade. I learned so much from sport through the highs and the lows. It has definitely shaped me into the person I am today and I think sport is a fantastic avenue to challenge yourself, reveal character, to develop you as an individual. Sport has matured me much faster than people my age and I'm appreciative of that. It wasn't easy but nothing in life that is worth fighting for comes easy. It has given me friends, brought me close to my family, given me a good work ethic and facilitated travelling the world.

The examination of these athletes' experiences demonstrates the highly complex relationship between the manifestation and nature of psychological symptoms and the stressors and benefits associated with competitive sport participation.

The effects of mental illness on sport performance. Highly related to the concept of mental health and sport being intertwined are the athletes' perceptions of the effects of their mental health challenges on sport performance. While this theme represents another example of how the athletes perceived their mental health and sport to be intertwined, it warrants its own category because of a conceptual difference with the examples outlined above. The theme "sport and mental health are intertwined" includes elements of competitive sport that the athletes perceived as impacting their mental health, whereas this theme describes how the athletes perceived their mental health to impact their sport performance. Indeed, the athletes shared many examples of how mental disorder symptoms manifested in the sport context, including fatigue 
and difficulty concentrating associated with anxiety, and low mood, social withdrawal, low motivation and anhedonia associated with depression interfering with performance.

Raevyn, Justin, and Heather described experiencing anxiety and they were able to reflect on how some of their symptoms affected their sport performance. For example, Raevyn constantly felt fatigued when she was anxious, which, not surprisingly, made it difficult to optimize her physical capability such that the effect was noticeable to her coaches:

Raevyn: One of my coaches pulled me aside one day after I had a really rough race. She pulled me aside and called me out for suppressing everything and told me that we needed to have a more aggressive plan to address the issues more directly. I was also sick of it hindering my performance. It was becoming the main part of why I was having bad races; just being constantly tired and having my life anxiety seep into my running. Another example of the relationship between anxiety and sport performance is Justin's experience with his inability to shift focus from his anxious thought interfering with his ability to focus and communicate with his volleyball partner:

Justin: The anxieties and worries I had about my relationships with people usually showed in some aspect in sport. If I'm nervous or have anxiety about something in my relationship with my girlfriend my focus shifts and I don't play as well. Or if you have anxiety about something on the court it usually comes out in the way you communicate with your partner. If I have an issue and I'm not telling my partner what is going on it's probably not going to help us get through stressful situations.

Similar to Justin, Heather described having difficulty at times keeping anxious thoughts from interfering with her ability to focus in training and competition. She also identified a general 
preference of being in a "good mental space" when she competed because she felt that it would enhance her performance:

Heather: I know I always perform better when I'm in a good mental space. I definitely think there is a lot of benefit to having good mental health in terms of performance. In my experience you can't play worse because you're feeling better. I am good at being like I'm just here to play and nothing else matters. Then the game is over and I'll think about it. I definitely try to divide those because I'm more productive when I'm just thinking about volleyball. But that's not to say that I've never had practices or games when I'm warming up and thinking about my worries.

The impact that Mia, Clara, and Pierre perceived their mental health to have on their sport performance was related to the symptoms of depression these athletes experienced. Mia described the effect that severe depression had on her ability to maintain focus and engage in the immense physical and emotional effort of a running race:

Mia: I did feel like my mental health was intertwined with my sport performance and health overall, and my mental skills for sport. At the absolute peak of suicidal depression, I went to a race and just bombed it completely. At the time I was like, "why?! I shouldn't have bombed it; it shouldn't affect my performance". But during the race I felt like I was in a dream, I felt like I wasn't even at the meet. I felt like I shouldn't have gone to the meet because I was so out of myself, just a complete shell of myself.

Evidently, Mia experienced a diminished sense of self when she was depressed because this state was inconsistent with her normal state of being. Being unable to maintain her performance expectations further ruptured her sense of self that was tied to being a successful athlete. An additional element of her experience with depression that Mia identified as interfering with her 
ability to engage in training was decreased motivation and social withdrawal: "I started to feel like my depression would affect my commitment and interaction with the team, and my mood at training." Clara's description of her low mood and amotivation when depressed echoes Mia's experience. These symptoms left her feeling unprepared for the effort required to complete difficult training sessions and maintain her training schedule, which ultimately contributed to worse symptoms:

Clara: I've had about seven injuries that have put me out for significant periods of time and I would still take any of them over the season when I was depressed. For me it's impossible to do this sport if you're not happy. I wasn't showing up mentally prepared at training so it didn't really matter what the workout was, I just kept leaving more and more defeated and down and angry with myself and depressed. That just translated into more physical injuries because I wasn't eating properly or taking care of myself. There have been times when I've been physically healthy but deeply depressed and it was such a struggle to get out the door for a run.

Finally, Pierre reflected on the disillusionment and decreased motivation he had within his sport and how it affected his ability to perform when he was experiencing the sport specific pressures and stresses that he perceived as triggering disordered eating and depression. His performance was impacted because of the psychological and physical paralysis that occurred when Pierre questioned the purpose of his dedication to competitive sport and became depressed:

Pierre: I started questioning the point of all of this, where the fulfillment in all of it was, what I was doing it for. When that started happening, I realized I was unhappy with what was going on in my sport, I didn't understand what I was doing it for and what I was 
gaining from it, I just didn't get it. It started creating this mindset where I would show up to practice and I couldn't move, I couldn't do anything.

Evidently the relationship the athletes perceived between their mental health and sport is multidirectional; they described elements of their competitive sport experiences that had both positive and negative influences on their mental health, and symptoms of their mental health challenges that influenced their sport experiences, namely their ability to train and perform in competition.

Athletes' Understandings of Mental IIlness Within the Context of Sport. Examining both these athletes' perspectives and perceived others' expectations of what being a competitive athlete entails reveals an assumption in competitive sport culture that complete commitment and dedication to sport performance and demonstrating superior psychological fortitude are expectations of this role. In comparing this normative discourse with the reality of the complex relationship that these athletes described between competitive sport participation and their mental health, and the immense impact their mental health had on their ability to perform in their sport, we can begin to understand how their experiences with mental health challenges within this context has shaped their perceptions of athlete mental illness. Based on this analysis, the athletes perceiving an incongruence between being a "tough" athlete and having mental illness, and that psychological factors are an unacceptable explanation for performance issues. A consequence of the internalization of these views is the self-stigmatizing attitude that mental health challenges are a weakness in the sport context and, for some of the athletes, this attitude has led to denial that their psychological distress may actually be symptoms of clinically significant mental illness. 
Having mental illness is incongruent with being a tough athlete. In the context of discussing the athletes' perceptions of their mental health and athlete mental health in general, many of them revealed an attitude that having a mental illness was incongruent with the normative image of a competitive athlete. Mia's reflection on her hesitation to disclose her diagnosis of depression with one of her coaches demonstrates this attitude:

Mia: I'm a very competitive person and I just told myself, "no, no you're tougher than that, you're an athlete, you're fine, you can train, you can do anything, you're so strong because you're an athlete." It was because of that athletic identity, that the depression doesn't matter. I didn't want to tell my coach because I didn't want him to go easy on me. I wanted it to be all business. In my coach's eyes I wanted to be tough and competitive. I didn't want him to see me at my weakest because I wanted to impress him. I didn't want him to treat me any differently or see me any differently because I always wanted to be his tough, good athlete, a good runner.

Heather and Justin also expressed concern that their mental health challenges would be perceived as a weakness within the sport context; a perception that was produced by the incongruence between the version of an athlete that was expected within their sport culture and expressing emotion:

Heather: For a while it was not something I talked about with my coach. When I did reach out to my coach, I said I had known this [experiencing anxiety] had been going on for a few years and the reason I didn't tell him was because I didn't want it to be a sign of weakness or something to be defined by. I think for all athletes when you compete for playing time, you're trying to be the best version of yourself and you need to be really 
competitive. Admitting that you need help when it comes to mental health issues definitely comes across as weakness in my mind.

Justin: I didn't want to be someone who hid their feelings in sport, but I always felt so many pressures from friends or people I was supposed to respect, like you're supposed to respect your coach. I think that's the macho culture, that you're supposed to be manly and that is being tough and not showing weakness.

Interestingly, Heather reflected on her internalization of this perception such that she viewed her mental health challenges as a disadvantage when it came to sport performance. Similar to Justin's identification of a "macho" male culture in his sport contributing to the perception of mental illness as a weakness, Heather described a gendered component to her experience:

Heather: People can talk about it being stigma or whatever but I'm going to be quite honest, would I rather compete with good mental health or bad mental health? That's not an advantage for me, right? I think my perspective is internal but obviously it has to come from somewhere. The way athletics is portrayed, there are a lot of stereotypes in sports that I get that message from. It's thinking that you have to be really tough if you want to be good at sports. People don't see mental illness as being part of someone who is really tough and really gritty. For me it also comes down to being a woman in sport. There are a lot of things women have to do to prove themselves that men don't have to do. If you want to succeed in sport you have to be tough and you have to be gritty. Considering that not all women are considered tough, like that's not a very feminine feature to start with, you have to do the little extra things to prove yourself. Admitting that you have mental illness is not one of those things that's going to help 


\section{Mental health challenges are an unacceptable explanation for performance problems.}

Related to the perception of an incongruence between being a tough athlete and experiencing mental illness was the attitude expressed by some athletes that mental health challenges were not an acceptable explanation for performance issues in training and competition. These athletes described experiences in which they defaulted to a plausible physical excuse despite internally acknowledging that psychological symptoms were likely more relevant. A salient example of this theme was shared by Heather:

Heather: In the middle of my practice I just started bawling my eyes out. I remember breathing really, really heavy. I had had exercise induced asthma before, and I just started crying and had this wheezing thing going on. I was saying, "it's asthma, it's asthma", but it wasn't asthma, it was definitely anxiety. That was the first time I realized I was struggling and maybe mental health was something I should actually be concerned about. Pierre and Clara discussed how they were aware of themselves engaging in this tendency to use physical issues as an excuse for performance issues because admitting that psychological factors were at play was perceived as less valid and would lead to judgement from others:

Pierre: There's a heavy emphasis on physical health, on injuries, because they are easier to see. Mental health can be challenging to manage and, in diving specifically, often presents as performance slumps. We have these top tier athletes who seem to be able to do everything and then all of a sudden they show up to practice and can't get their bodies to perform acrobatic movements that they used to do. The language around it is that they are going through a slump.

Clara: The excuse or reason for a performance issue is always easier to express if it's physical than if it's mental, but I think more often than not it actually is more mental for a 
lot of us and we just don't know how to express that. I've dropped out of workouts before because of mentally doubting myself but then I make the excuse that something hurts. In the same sense that you can have a stress fracture and that can be the reason not to race well, it felt like I didn't have the tools to access the fact, even to myself, that maybe what's going on is actually a mental health issue.

Clara also spoke of her awareness of other competitive athletes hesitating to acknowledge mental health-related explanations for performance issues, and what a powerful message it would have been for her self-acceptance if they did more often:

Clara: I would love more runners to be able to say, “I didn't race this season, not because I had a stress fracture, but because I was so anxious, or I was so depressed.” That's not really something that is said, it's still so stigmatized. You watch athletes get into races and drop out and they don't really have a physical reason, but it's so rare to see someone post something like, "I had to drop out of this race because I had anxiety, or a panic attack." I would have so much respect for someone saying that, but there is a trend to look for any exterior excuse to mask what's going on.

These athletes described a shroud of silence surrounding mental health issues in sport that evidently served as an impediment to disclosure and, as we will examine later, interfered with accessing treatment. Finally, Mia reflected on how this perception actually shifted for her once she underwent treatment for depression, but her original attitude is highly indicative of this theme:

Mia: When I was a younger athlete I felt like my depressed thoughts, or when I was in a low mood, that it should not affect races. I should be able to go to every practice, every 
race, and my muscles are separate from my brain, it doesn't matter. As I grew I did realize that it's okay for mental health to impede your performance.

Self-stigmatization and denial. Thus far we have explored the disconnect between the normative understanding of what it means to be a competitive athlete produced by competitive sport culture, the reality of the lived experience of an athlete with mental illness, and the effect of this incongruence on these athletes' understandings of mental illness in the sport context. Consequences of this incongruence are self-stigmatization and denial of the severity of mental health issues by these athletes. For example, Pierre acknowledged that societal expectations of the characteristics of competitive athletes creates pressure to ignore significant psychological distress. He also identified the potential for the characteristic of resilience, which is typically valued in competitive athletes, to work against an athlete when acknowledging distressing psychological symptoms may be necessary:

Pierre: I think a lot of athletes go through these types of challenges [mental health challenges] and don't realize they're going through it because the personality of athletes who get into elite sport is to be resilient and push through most things. The nature of that personality is maybe that they will feel depressed or upset and just think it's normal, they just accept it.

There was considerable nuance in how the athletes perceived stigma. Athletes described experiencing both perceived other and self-stigma, which is the internalization of perceived societal attitudes toward mental illness in sport such that the athletes endorse stereotypes about themselves (Corrigan \& Rao, 2012). In a representative example, Clara described how she notices herself endorsing the stereotypes that she perceives others as having about athletes who express psychological distress: 
Clara: When it comes to the stigmatization of mental health, it is starting to get better but I'm guilty of it too, you know, thinking in my head of teammates who get dramatic sometimes. I might not say this out loud, but in my head I'll be like "oh they're such a head case". I should also add that stigmatization isn't just from what other people think of you, but it's on myself too. I don't want to be someone who always has an excuse, or always is talking about something depressing. I want to be acting in a way that reflects who I think other people perceive me to be or I'm told other people perceive me to be. In addition, Pierre's internalization of the stereotype that mental illness is a personal weakness in the competitive sport context led to him feeling shame and internally denying his symptoms:

Pierre: I think for a while I didn't really understand what my depression was. I think a lot of that is the stigma around mental health that makes you never want to admit that you're depressed because it's seen as a weakness. Athletes don't want to show weakness. There's that internal competition that you don't want to make yourself vulnerable by explaining that you're having a hard time. There is also a level of embarrassment. I started to realize that it was not normal, and deep down I knew it was depression, but I was so reluctant to admit that to myself. There was complete denial the entire time. Another contributor to the stigma that the two male athletes, Pierre and Justin, described was related to the gendered concept of a "macho" culture within their sports. For Pierre, the pressure he felt to conform to behavioural expectations of a normative male athlete led, in part, to the denial and suppression of his sexuality and severe self-policing of his behaviours, which he perceived as contributing to his depression symptoms:

Pierre: Simultaneously with my period of questioning the purpose of pursuing my sport, I was struggling with my sexuality and trying to internalize that, trying to come across as 
macho. But my sport was very public, everyone sees you, they see your body language. I was so calculated in how I behaved, how I moved my hands, how I spoke, because I didn't want anyone to assume I was anything but straight. I was internalizing everything and not talking about it. It turned into an overwhelming depression that I just didn't know how to manage.

Justin also identified the role of conforming to a "macho" male culture in his sport, which dictated that expressing emotions was unacceptable, in increasing stigma toward experiencing psychological distress:

Justin: In sports with macho cultures, especially contact sports like hockey, there's an incredibly large physical component to it. I think from the physicality and being tough you're not supposed to show weakness. So, with that, it's like if you feel emotions, hide them because that's weak if you show them. You're not going to be respected as an opponent if that happens, so you're taught to not cry if you're frustrated.

Many athletes also reflected on the state of stigma toward mental illness in sport, specifically they perceived that although it is improving, it is doing so at a slower rate than in general society. For example, Justin reflected on the changes he has noticed in the acceptability of emotional disclosure in sport and why he believes the stigma in sport culture is worse:

Justin: I think athletes are put on too high of a pedestal to begin with and that forces you to put on this face all the time. I think the world around sports is changing a lot faster than the world within sports, I mean talking about mental health is becoming much more prominent at a faster rate than it is within sports. I just think sport doesn't provide enough tools to understand how to do that. It's changing, I do see it changing, but it could change a lot faster. 
Similarly, Clara reiterated the perspective that stigma is improving but that the culture of competitive sport is behind: "I think it's getting better but it's definitely something that hasn't been nearly as accessible or talked about in competitive sport as it should be, or that it could be."

Finally, Brenda had the unique opportunity in this group of athletes of being able to compare her perception of stigma toward athlete mental illness as an adolescent engaging in competitive sport in the 1990s and her experience as a current competitive Masters athlete and coach:

Brenda: I grew up in an era where everything was hush hush, you don't talk about that kind of thing [mental illness]. It was so stigmatized back then, and I mean it still is, but it was worse back then. I think things are changing for the good, but when I was growing up you were called crazy if you had any sort of mental health issue. You would never mention anything to your coach about it. I think people are starting to view it more as another medical thing, and I think it's much more acceptable to be open about it.

Through exploring and developing an understanding of how these athletes perceive the role of a competitive athlete, how they perceive the relationship between their sport participation and their mental health, and finally how they perceive mental illness in the context of sport provides rich context for examining the next two research questions regarding athletes' experiences with mental healthcare.

\section{What are Athletes' Perspectives on the Importance of their Mental Healthcare Provider}

\section{Having a Specific Understanding of the Sport Context?}

To provide context for this question, it is helpful to understand what kind of mental health care providers the athletes worked with and the nature of their sport experience, based on what was reported by the athletes. This information is found in Table 2. Some of the athletes 
discussed experiences working with a "sport psychologist" on mental skills and performancerelated issues. These individuals are not included in the table because they did not treat the athletes for mental health-related concerns, and all had extensive sport-specific knowledge as a pre-requisite to their profession. At times the athletes' reflections on their experiences working with these individuals are included in the following themes because they do help us understand why some athletes considered working with a professional with sport knowledge as being beneficial. In terms of addressing the research question, it was clear that the perceived sport specificity of the mental health concerns for which they were seeking treatment influenced the importance athletes placed on their mental healthcare provider understanding the sport context. This finding is described in the first theme, "Sport specificity of mental health concerns". Furthermore, the athletes reflected on reasons why knowledge of the sport context was relevant to psychological treatment, which are outlined in the themes "Normalization and validation" and "Understanding the demands of competitive sport culture". The final theme in this section, "Other components of a successful therapeutic alliance" reflects the aspects of psychological treatment and characteristics of treatment providers unrelated to having specific experience with sport that the athletes perceived as important for a therapeutic relationship and ultimately effective treatment.

Table 2.

Mental healthcare providers and sport knowledge

\begin{tabular}{|lll|}
\hline Athlete Pseudonym & Mental Healthcare Provider & $\begin{array}{l}\text { Provider Sport } \\
\text { Knowledge }\end{array}$ \\
\hline Brenda & Clinical psychologist & None \\
\hline Heather & $\begin{array}{l}\text { Clinical psychologist and } \\
\text { counselor }\end{array}$ & None \\
\hline Justin & Clinical psychologist & None \\
\hline Addison & Clinical psychologist & None \\
\hline Jasmine & Clinical psychologist & $\begin{array}{l}\text { Experience working with } \\
\text { competitive athletes }\end{array}$ \\
\hline
\end{tabular}




\begin{tabular}{|lll|}
\hline Clara & $\begin{array}{l}\text { through affiliation with } \\
\text { sport organization }\end{array}$ \\
& $\begin{array}{l}\text { Psychiatrist; counselors; Sports } \\
\text { medicine physician }\end{array}$ & $\begin{array}{l}\text { Psychiatrist and counselors } \\
\text { (none); Sports medicine } \\
\text { physician (experience } \\
\text { working with competitive } \\
\text { athletes through affiliation } \\
\text { with sport organization) }\end{array}$ \\
\hline Mia & $\begin{array}{l}\text { Clinical psychologist; family } \\
\text { physician }\end{array}$ & None \\
\hline Emma & Clinical psychologist & None \\
\hline Raevyn & $\begin{array}{l}\text { Counselor; sports medicine } \\
\text { physician }\end{array}$ & $\begin{array}{l}\text { Counselor (none); Sports } \\
\text { medicine physician } \\
\text { (experience working with } \\
\text { competitive athletes } \\
\text { through affiliation with } \\
\text { sport organization) }\end{array}$ \\
& & None \\
\hline Pierre & &
\end{tabular}

Sport Specificity of Mental Health Concerns. Based on the nature of the specific mental health challenges that the athletes faced, and the nature of their individual relationships with sport, their perspectives varied on how related their mental health was to their sport experience. This relationship, in turn, consistently overlapped with how relevant they believed a mental healthcare provider's sport knowledge was to their treatment experience. Justin and Clara's contrasting experiences highlight each side of this perspective:

Clara: If I wanted to speak directly about the medication I was on for anxiety and depression maybe they wouldn't need to be familiar with sport, but I just don't really see any conversation that wouldn't tie back to running to some extent because that's the main focal point of my life right now and what everything has revolved around, the whole lifestyle that we were talking about. I think either way it would be beneficial, even if it wasn't something that I could pinpoint to affecting my running. 
Justin: At the time it wasn't directly related to sport [reason for seeking mental health treatment]. A lot of my issues that I dealt with in therapy were related to times when I was like nine years old to 16 years old. I didn't feel like it was super relevant to my treatment because I knew a lot of the issues that were causing anxiety were related to my relationships with my family members and people in my life growing up. That's what a lot of it was. So to be honest it [sport] wasn't coming up too much at the time and I wouldn't be able to assess how well the therapist knew my sport.

In Clara's case, she perceived her experience with mental illness as being inextricably linked with her sport, that sport was both a coping mechanism and a source of psychological stress. She also described having a strong athletic identity and her lifestyle being structured to facilitate sport performance:

Clara: Overall my experience has been very lifestyle changing, really all inclusive. I don't want to say that everything comes secondary to running...but my life seems to be planned around training now. It's a 24 hour job in a sense. A lifestyle too in the sense of the people I'm surrounding myself with. I've lost the tightness of the connection with friends that have nothing to do with the sport, our lifestyles were drastically different. Raevyn echoes this perspective, that being an elite athlete means that many aspects of an athlete's life and identity orient around this pursuit, making it very relevant for a mental health care provider to understand the sport context:

Raevyn: I do think whether people believe it or not, your real-life ties into your sport and you can suppress it all you want but if you can't tackle your real-life issues it's connected to your sport. So I think it's important. Especially when people prioritize it [sport] and it's the biggest part of their lives. And the idea that people put their self-worth in their 
sport and their results, I think when people are battling that kind of stuff it does tie so much into their real lives. I think counsellors or psychologists would have to understand that... and why it's important, that it's not just as simple as saying just don't put your identity into it.

Finally, Pierre perceived the stressors he experienced in his sport culture to be causally related to his mental health challenges and therefore felt strongly that his mental healthcare provider should understand how significant an influence sport can have in a competitive athlete's life. When this understanding was not conveyed by a psychologist at his university's health centre the experience was so invalidating that he never accessed care again:

Pierre: I met with a counselor and spilled my guts about everything that was happening. I was so upset and said I needed help and the response I got was the opposite of what I needed. I don't know if I shocked him or if he wasn't prepared but he seemed very confused, almost like he didn't understand what I was upset about. He said things like, 'well you're still doing well in school, you have a family that loves you. You can't compete in your sport but you have all these other things that are fine'. I was like, "What?! Ya okay but those aren't concerns, my life is this sport and I've been robbed of my ability to compete and that's what I value and you're basically telling me that what I'm feeling isn't true". I was so put off by that. I wasn't in a state to hear that and it wasn't okay. It left me feeling more discouraged, and that was my experience with professional care.

On the other hand, and similar to Justin's perspective, Mia describes a very different relationship with her sport, mental health and identity. Mia trained and competed at a national level in her sport and described her involvement as, "pretty intense, it was a big commitment and 
I'm very committed". However, she attributed her experience with depression to stressors outside of sport and therefore the knowledge her psychologist had with the sport context was less relevant to her treatment experience:

Mia: For me it was enough [that the psychologist just valued my sport participation]. My life is so much more than sport, so I only talked about it as maybe $10 \%$ of the conversation. My mood would also affect my relationships, my studies, my family, my everything, self-worth. It was much more about life as a whole and sport as just a part of it. But for someone who sport is their whole life it may be more important.

Emma shared this perspective when she discussed the lack of importance she placed on her therapist's sport-specific knowledge:

Emma: I wasn't aware of how much of an impact sport had on my eating disorder. I wasn't attributing the problem to sport at the time. Maybe if I had been more aware of the link I would have wanted someone with more sport expertise, but honestly at the time I just wanted someone to talk to so it didn't really affect the experience at all.

The athletes who did view their mental healthcare provider's knowledge of the sport context as an important factor in their treatment experience discussed why specifically this knowledge was beneficial. As mentioned, these themes are also informed by the perspectives of athletes who worked with a mental skills trainer on sport performance-specific issues or a sports medicine doctor in addition to a clinical psychologist or psychiatrist. While the mental skills trainers athletes worked with were not treating mental health concerns, many of the athletes discussed their mental health with these individuals and reflected on how these counselors' extensive experience in the sport context enhanced their ability to form a therapeutic relationship. These concepts are elaborated on in the following two themes. 
Normalization and Validation. Working with mental healthcare providers or mental skills trainers with experience in the sport context was perceived as beneficial because of their ability to validate and normalize the athletes' experience with mental illness. As we learned in our exploration of the elements of competitive sport culture that are related to athlete mental health, the athletes in this study perceived an incongruence between being a tough and successful athlete and having a mental illness. This perception contributes to the assumption that most high performing athletes are not struggling with mental illness, and therefore feelings of isolation and inferiority when an athlete does find himself or herself in this situation. Hearing from an individual who has had exposure to a large number of athletes seeking support for psychological issues, whether in the realm of mental illness or sport performance, was a powerfully validating and comforting experience. For example, Raevyn felt ashamed of taking medication to treat her anxiety and depression until she learned from her sports medicine doctor that many other athletes have done the same:

Raevyn: I felt very alone in the fact that I was on medication. Until my doctor told me something that really helped me, which was that most athletes are dealing with something like this and he has prescribed medication for other people. It was really cool to hear that because I thought that I was just going through stuff and everyone else was killing it and doing well, which was obviously kind of ignorant.

Similarly, Mia reflected on how reassuring it felt to have her experience with depression and suicidality normalized within the sport context, and Pierre described why having his experience normalized would have been reassuring if he had been able to access a care provider with sport experience: 
Mia: He [mental skills trainer] said he worked with a lot of professional athletes and that he hears about these issues [depression and suicidality] a lot. It felt good to know that other athletes feel this and they can still be successful and work through it. He was able to tell me that feeling this way is normal, that it sucks but it's normal. He just accepted it, as if I had said "I got a stress fracture this year", like it's just another thing that can happen. Because he knows so much about sport he really validated my experience of my mental state affecting my sport. It helped me realize that I'm not crazy. When he said a lot of athletes go through that I instantly felt a little bit of light, like a little ray of light.

Pierre: I think it would be so valuable to work with someone who does understand sport. I think it would be valuable because of their ability to support athletes and be able to tell them that it's okay, that what you're experiencing is normal for what you're going through. That was one of the biggest things for me, just hearing from people that it's okay, because I felt like I shouldn’t feel this way.

Finally, Jasmine's perspective reiterates the benefit derived from learning from a mental healthcare provider with experience working with other competitive athletes that mental health challenges are common in this population despite culturally-based assumptions:

Jasmine: It was helpful knowing that she [clinical psychologist] does work with a lot of top athletes in the region and having her say things like, "A lot of athletes I work with will bring up similar issues'. Obviously, she maintains confidentiality, but she'll talk about how athletes have experienced something similar.

Understanding the Demands of Competitive Sport Culture. For the athletes who perceived their mental health to be highly related to their sport experience, their mental healthcare provider understanding the demands and nuances of competitive sport culture was an 
important component of effective treatment because it facilitated and strengthened the therapeutic alliance. For example, Addison and Pierre felt that it was important for a psychologist or mental skills trainer to have a level of personal experience with sport to understand the pervasiveness of the competitive athlete lifestyle:

Addison: I find it's related to the level they played at. If you're doing a sport for fun you don't have that same, "Oh my gosh this is so important to me" understanding. My sport psychologist played at a really high level in her sport so she can understand there's that added pressure once you decide to become an elite, and just how much it translates into the rest of your life.

Pierre: I do truly think that that is absolutely necessary [clinician having sport experience]. I just think that competing at sport at that level is so unique and you can't really get it until you've done it. They need to have some sort of relevant sport background to understand where these athletes are coming from, why they feel their everything is their sport. I think the counselor I spoke to was genuinely so confused. People look at sport like it's not important, like how could it be distressing? They just don't understand the cultural pressures of it.

Similarly, Clara discussed understanding the psychological demands that competitive athletes experience as a helpful component of the relationship she was able to establish with a mental skills trainer she worked with:

Clara: The fact that she [mental skills trainer] had a background within sport and understood the demands that I was going through was really helpful. I think that that's something that's so important with any psychologist working with an elite athlete, that they have at least some experience or understanding of what it takes and the mental side 
of how exhausting and demanding all of these thoughts that go through our heads are, that may not go through the other average patient's head.

Jasmine also believed that her psychologist having exposure to the elite athlete lifestyle through treating other athletes or having personal experience with sport was important in her treatment experience, in this case because it was comforting and made her feel understood by her therapist:

Jasmine: I feel that she [the clinical psychologist] has a strong understanding of the life of an athlete. That has really been an asset and makes me feel less alone and more understood because she knows how my lifestyle has been thus far. She seems to have a really solid grasp of that and that in itself is really comforting. I think it's so hard to articulate and share the lifestyle of how you live as an athlete. I think it's kind of hard to understand if you haven't been exposed to it before. There are so many elements that people wouldn't even think of.

Jasmine evidently felt that it would be difficult to explain the elements of competitive sport culture that many of the athletes perceived as being intertwined with their mental health with a mental healthcare provider with no exposure to the sport context.

Finally, this theme is reflected in Raevyn's reflection on her experience working with a counselor who did not have experience working with high level athletes. She perceived the counselor's lack of understanding of the nuances of competitive sport culture as interfering with her ability to establish depth in the therapeutic relationship:

Raevyn: I wish I could talk to my counselor about running, but it didn't really come much into conversation other than surface level stuff or just the fact that I train a lot and have goals. If she did realize how big a part of my life it was I felt like it just got blanketed to everyone who does high level sport. So I found myself not really going 
there. I would go there a little bit, but it would just make the conversation about my life pretty general.

Other Components of a Successful Therapeutic Alliance. The reality of these athletes' experiences is that few had access to mental healthcare providers who had specific experience working in the sport context. Therefore, I considered the value of exploring the athlete's ideas regarding what contributed to successful treatment experiences despite their provider not having sport-specific knowledge. As described, some believed this was detrimental to the relationship they were able to establish with their provider. In contrast, athletes who perceived their mental health challenges as more highly related to domains outside of sport were satisfied by other provider characteristics that contributed to rapport building. From Emma's perspective, the athlete who did not connect her eating disorder with her sport experience, having an outside perspective was even viewed as an asset:

Emma: At the time it didn't hinder or help in any way that they commented or didn't comment on sport. To be honest at the time I didn't really think about it. I think it's probably valuable to see both sides. I don't think she necessarily had to have expertise in my sport, but maybe just sport in general and having an idea of the athlete mentality would be helpful. But I also like the outside perspective because they see people from all different backgrounds with these issues, so maybe they have a more global understanding of eating disorders. I didn't think it was bad that they didn't have a sport background. Regardless of having personal experience with sport or with working with athletes, a highly consistent perspective of this group of athletes was that the mental healthcare provider clearly conveying that they valued the prominent role that sport played in the athlete's life was sufficient to establish an effective relationship. Mia described this concept in the following excerpt: 
Mia: I definitely talked to my psychologist about sport. I actually asked her if she had any experience with sport psychology and she said no, not really. But she also said she felt like she could understand sport on a basic level and so when I would talk about competitive stuff, racing, or team dynamics and emotions I experience in sport she understood. She definitely did her best to understand and remember, like when I would talk about different race distances or events she would remember. She was really good about it and she definitely tried to understand how depression affected my running. Her being open to talking about sport and trying her very best to understand it was great. Sharing my experience with sport with her just helped her relate more to me as a person and just build that stronger rapport.

Brenda felt similarly, that having skills in conveying empathy and an effort to relate to her experience as an athlete created a sense of safety and trust in the relationship even though her psychologist did not have experience with sport:

Brenda: She [psychologist] wasn't an athlete herself. Normally I think unless you're an athlete that you wouldn't really understand that, but she actually seemed to get it pretty well, she was pretty empathetic. I think it's all about finding someone who can just relate to you and normalize your feelings and understand them to a certain degree.

Heather's experience echoes the comfort that Brenda derived from an empathetic therapist. She also identified that forming the impression that their mental healthcare provider was intelligent, experienced, and qualified made her feel comfortable and secure in the therapeutic relationship: Heather: I could tell talking to her that she was super smart. So that was definitely really comforting. She definitely knew what she was talking about and was good at getting people to open up without feeling like she was trying to make people open up. So it was 
nice to have her to just talk to. It flowed out of her without her being cocky about it, there was no sense of arrogance. It was comforting, I knew I was in good hands. So maybe having somebody who is a little bit more sport trained would be good, but I really can't complain.

Addison's perspective incorporates both of these components; both her impression of her psychologist's professional expertise and the fact that he conveyed his value of the role of sport in her life were sufficient to effectively work together:

Addison: When I walked into his office and I saw all these books and journals it resonated to me that he cared about what he did. He did research about what was important to his practice. I think the reason I was confident with the psychologist was that he was a master at what he did. He really cared about how important sport was for me and didn't judge me for it becoming a bigger part of my life at that time. I think they just can't be anti-sport; they can't be closed off to the therapy of sport itself or feel like it's a waste of time. I think it would be poor to engage with someone like that. I don't think it always matters [to have specific sport experience]. I think if the person is relatable that's the most important thing. If you don't have rapport it doesn't matter how much they know about your life or your world.

Therefore, the themes in this section reflect the nuance required to understand the importance that these athletes placed on their mental healthcare provider having sport-specific knowledge. Essentially, the athletes who perceived their mental health challenges to be highly intertwined with their sport experience had difficulty connecting with mental healthcare providers who did not have sport-specific expertise. For those athletes who attributed their mental health challenges to domains outside of sport, such as relationships and life transitions, 
in-depth knowledge of the sport context was not considered a critical factor in establishing an effective therapeutic relationship. Rather, conveying empathy, valuing the role of sport in the athlete's life, and conveying an impression of intelligence and professional expertise were sufficient for the athletes to establish rapport with their mental healthcare providers. Finally, athletes who did have experience working with a mental health care provider or mental skills trainer with sport-specific knowledge identified the ability to normalize and validate their experience within the sport context, and to understand the psychological and lifestyle demands on competitive athletes, as the beneficial aspects of having sport-specific knowledge.

\section{What are the Avenues by Which Athletes Access Mental Healthcare, Including the Barriers and Facilitators to Accessing Care?}

Based on their experiences accessing mental healthcare, the athletes were able to discuss the avenues by which they received care and their perceptions of the barriers and facilitators to both help-seeking and ultimately accessing treatment. The themes discussed in relation to this research question shed light on the types of venues at which a group of competitive athletes in Canada are accessing mental healthcare and on both the individual and cultural influences that play a role in inhibiting and facilitating this population seeking mental health treatment.

Venues of Mental Healthcare Accessed by the Athletes. The majority of the athletes accessed mental healthcare from the private sector. A handful of the athletes were university students at the time that they accessed care and therefore sought services through their campus health centres. Mental healthcare was also accessed through publicly funded sports medicine and family physicians. Table 3 includes an overview of where each athlete accessed mental healthcare.

\section{Table 3.}

Athletes' venues of mental healthcare 


\begin{tabular}{|ll|}
\hline Athlete Pseudonym & Venue of mental healthcare \\
\hline Brenda & Private practice \\
\hline Heather & Private practice and university health centre \\
\hline Justin & Private practice \\
\hline Addison & Private practice \\
\hline Jasmine & Private practice \\
\hline Clara & University health centre and doctor's office \\
\hline Mia & $\begin{array}{l}\text { Private practice, university health centre, and } \\
\text { doctor's office }\end{array}$ \\
\hline Emma & University health centre \\
\hline Raevyn & University health centre and doctor's office \\
\hline Pierre & University health centre \\
\hline
\end{tabular}

Facilitators of Seeking Mental Healthcare. This theme encompasses the influential individuals who encouraged the athletes to seek professional treatment for their mental health challenges and the characteristics of their environments that made them perceive mental healthrelated help seeking as acceptable.

The coach-athlete relationship. Particularly when an athlete's mental health challenges manifested within the sport context, coaches were identified as individuals who facilitated helpseeking behaviour because of their role in noticing mental health-related symptoms or performance effects and suggesting treatment. For example, as we learned in the earlier discussion of the effect that Raevyn's symptoms of anxiety had on her sport performance, her coach initiated an intervention to address the issue after a string of uncharacteristically poor performances:

Raevyn: One day I had a really rough race in the indoor season and my coach pulled me aside. She called me out for supressing everything and told me that we need to have a more aggressive plan to address the [psychological] issues more directly. Since then my coach has encouraged me to see a counselor more often. She wants me to seek more specific treatment for first and foremost my health as a person and also my performance. 
Similarly, Pierre's coach was the first person in his life to directly address the changes in his behaviour associated with depression and disordered eating and encouraged him to seek treatment:

Pierre: I finally reached out to my coach. I started making excuses that I couldn't come to practice. I was so depressed I couldn't physically get out of bed. Eventually my coach reached out and asked me to come over so we could chat. I think that I was screaming for someone to notice because I didn't know how to ask for help. That was the lifeline that I needed. He was very adamant that he wasn't trained in how to do this but that he wanted me to first tell my parents that this was happening. Then he encouraged me to seek professional counseling. Did it save my life? Probably.

Heather's coach also encouraged her to seek mental health support when she confided to him the distress she was experiencing as a result of competitive team dynamics and balancing the demands of being a university student-athlete: "I had a rough patch during the winter. I told my coach about it and he directed me toward our therapist here at the university."

In addition to coaches explicitly encouraging the athletes to seek mental healthcare, the more general nature of the coach-athlete relationship affected athletes' comfort level in seeking support. When the coach-athlete relationship was characterized by acceptance, understanding, and open communication about sport and non-sport related stressors, athletes felt more comfortable disclosing their mental health challenges and drawing on their coaches for social support within the sport context. This coach-athlete dynamic made athletes feel supported and helped them accept their mental healthcare needs. Clara's perspective on her relationship with her coach reflects how their dynamic facilitated her decision to access care: 
Clara: My relationship with my coach now is way more open and honest and I don't feel nearly as judged or required to make something up when my mental health is interfering with my ability to train and perform. Having a coach who can understand that and give you the idea to look for resources makes a big difference. He would just be like, 'hey I'm sorry you're feeling like this, it obviously isn't my area of expertise but let's see what we can do to find resources to help you.'

Heather and Jasmine described how the support they felt from their coaches to both access mental health treatment and, in Heather's case, to be accepting of the anxiety she experiences in the sport context, was comforting:

Heather: My coach was really good about it. When I told him that I was so mad at myself because I had missed practice because of being stressed and sleeping through my alarm, he told me no one on the team was mad at me or resents me. He said he knows this happens and it's okay. He was really supportive, I'm so thankful to have him as a coach. He has done a lot for me in that kind of sense.

Jasmine: I'm sure it [seeing a clinical psychologist] was with the support from my coach. I'm sure she's an advocate for looking after your mental health. I would mention it to her every once in a while that I had gone to see the psychologist and she seemed definitely supportive of that."

Mia discussed how being open about her experience with depression with one of her coaches was an important part of her process of self-acceptance and recovery because it led to feeling supported in the sport context: 
Mia: It was nice to talk to the head coach of my team because he has seen a lot of athletes go through it [mental health challenges]. It felt good to talk to him because he's so directly in the sport so I could get a bit of support from someone in that group.

Finally, Brenda was in a unique position in the group of athletes in this study to be able to reflect on the coach as a facilitator of mental healthcare access because she is both a competitive runner and coach in youth sport. When discussing her perspective on the role of the coach in supporting athletes' mental healthcare needs Brenda drew on her experience as a coach to convey her opinion that coaches have a responsibility to directly address overt signs of mental illness with athletes and connect them to appropriate resources:

Brenda: During the season if you identify anyone who you think is struggling, coaches need to have that conversation with them. My experience has been that whenever I have had to do that it has always gone well and has been appreciated by the athletes. Sometimes we think it's going to be taken so badly, that they're going to be mad, but every time I've had to bring something like that up with an athlete the response has been, "Oh thank god someone noticed". It's something as a coach you just can't ignore. If you see someone dropping weight all of a sudden for no reason you cannot ignore that. You have to bring it up. So many coaches are afraid to or think someone else is bringing it up, but often times everyone is thinking that same thing, that there must be someone on top of it when actually there is nobody.

Supportive and accepting cultural environment. A supportive and accepting environment was a facilitator of care because many of the athletes described the culture of their immediate environment, whether that was within their sport, university, or broader social context, as influencing their perception of the acceptability of mental health help-seeking. For 
example, noticing that other people in their context were sharing experiences of mental health challenges and seeking treatment, receiving encouraging messaging about mental health helpseeking, and having access to information about mental health and treatment resources all contributed to athletes feeling more comfortable seeking support.

Exposure to and therefore awareness of shared experiences with mental illness was a salient component of athletes perceiving their environment as being accepting because it normalized their experience with mental health challenges and help-seeking. For example, learning about the experiences of highly successful elite and professional athletes contributed to Brenda feeling that her experience with depression and anxiety was normal and acceptable in the sport context:

Brenda: I think a big part of it is actually more openness from role models, from the athletes who have spoken up about their own mental health struggles. For example, the ones I can think of are from the books I've read: Silken Laumann, her book is fantastic and about her battle with depression; Clara Hughes; Amanda Beard the swimmer; Dominique Moceanu the gymnast. I think I've read almost every athlete biography and I would say $85 \%$ of them mention that they've dealt with depression. I honestly think just having role models in sport that have been very successful and that are considered "normal", whatever that is, and have dealt with their own struggles, it makes it more normal for an athlete to think, "Well if Michael Phelps has been depressed then maybe I don't need to be ashamed of my feelings right now."

Brenda and Pierre also reflected on the benefits of learning about other athletes' experiences with mental illness; that viewing others modeling being open about their challenges may help other athletes recognize signs of mental ill-health and seek help: 
Brenda: Sometimes you find that if one person asks for help and is open about it then others start to be open about it too. I follow a lot of running Instagram things and one girl was open about having an eating disorder and then another one will say, "Thank you for sharing, I've dealt with this too." I think the more people who share and the more people who are open about it, the more good can come out of it because more people get help. Pierre: There isn't a lot of dialogue between athletes. For the people who have spoken out and been like, 'I'm struggling with this too", I would have been like, "Thank god, I have problems with this as well." But no one wants to talk about it. My coach encouraged me to talk to another teammate I had who was very successful. I did and it turned out he had gone through a lot of similar stuff. Just hearing from someone else and seeing that he worked through it was amazing. That was so encouraging, and just knowing that you're not alone.

Learning about friends' experiences with mental health challenges both within and outside of the sport context and being aware of more discussion of mental health in society in general, facilitated positive help-seeking attitudes for Raevyn and Emma. Knowledge of shared experiences reduced the negative associations Raevyn and Emma had with their own mental health concerns:

Raevyn: I'm pretty open about mental health stuff now. I didn't used to be, I used to be really embarrassed by it, but in the past year or two I've been more open. It helped seeing my friends go through it and realizing that so many people are affected by it; almost everybody has something. So I'm pretty open about that, that's not really an issue for me. Emma: I do think talking about mental health is much more public now. Even now on Facebook you see people posting their personal stories. I wouldn't do that but it makes 
you feel more comfortable knowing how many people do that. When I was growing up no one talked about it and I felt so much guilt and embarrassment. People are talking about it so much more and when I became more comfortable talking about it to friends and family I finally realized, "Wow someone else gets it".

In addition to learning about shared experiences facilitating positive attitudes toward help-seeking, receiving encouraging messaging about mental health help-seeking, and having access to information about mental health and treatment resources made athletes perceive their environments as accepting and supportive. For example, Raevyn identified the encouragement she received within the academic department of her university to address mental health concerns as contributing to an accepting culture that normalized this behaviour:

Raevyn: My school is pretty good about being really open with mental healthcare and stuff and I have professors who mention it often and are really understanding with how kids struggle, so I think that was something I was lucky to have. Even just how accepting the culture of my department is. So it just seems like the natural thing to try out [mental healthcare]. But ya, I guess it could have been more suggested through my athletics department because I don't think it is... it was more through the academic side that suggested I do it.

Justin and Heather were also aware of their universities' mental healthcare resources and thought that the visibility of such services was an important component of facilitating athletes' access:

Justin: I see at my university the effort to...like we have sport psychologists on staff with us, and we have lots of counselors you can go talk to. I see organizations and companies make more of an effort to reach those services out to athletes. It totally was offered and visible, which is important. 
Heather: At the beginning of the year the athletic department had an athletic retreat. One of the activities was different stations of all the resources that are available to athletes; one was the athletic therapist and how to book an appointment to get physio, one was academic support, and one was counseling and well-being. So I knew she was available to me. So about a year later I looked her up on my own and told my coach I thought I should make an appointment and he was like, "Ya I think that's a good idea'.

Finally, Mia and Emma discussed the role of an accepting environment that emphasized awareness in reducing the stigma of seeking mental healthcare and thus made them feel like it was a viable choice:

Mia: By the time I became depressed again I learned a lot more about mental health over the years from university seminars, courses, reading, the general public, media, stuff like that. So I felt a bit more proactive and went straight for the help. We're lucky we're in an environment and university and city where there's a little bit less stigma in general, so that's good.

Emma: No one specifically suggested I seek treatment, I just decided on my own. I guess they kind of promoted it throughout the school if that counts as encouragement. There are so many more resources advertised for this kind of thing, so if I wanted to see a counselor now I would have a much easier time just going and getting over that whole stigma.

Family and other influential individuals. The athletes identified other influential individuals who facilitated their access to mental healthcare by encouraging them to seek treatment. Specifically, family members, friends, teammates, and romantic partners suggested that the athletes pursue professional support when they noticed psychological distress. For example, Raevyn and Heather turned to their mothers for support who in turn helped them access 
mental healthcare. Raevyn said, "It was my mom mostly who told me about getting help from a psychologist. My mom told me it was a good idea."

In Justin and Jasmine's cases, their family members further facilitated access to mental healthcare by assisting with the expense of private psychological treatment. Jasmine explained that she was able to use her parents' insurance coverage to pay for her treatment and Justin's parents paid for his treatment:

Justin: In Canada I know the wait times are really long for public treatment. I was very lucky that my girlfriend forced me to own up to my parents about it before I sought treatment because I was not going to be able to afford an hour and a half of psychotherapy every week. It's super expensive. I was lucky my parents were in a financial position to be able to afford it.

In this excerpt Justin highlights the role that his girlfriend played in encouraging him to seek care. Raevyn and Clara also identified individuals other than family members, namely a close friend and a teammate, who knew these athletes well enough to identify that they were experiencing mental health challenges and suggest care providers that they had previously worked with. For example, Clara described the support she received from a teammate:

Clara: I started seeing a doctor at my university who I accessed through a teammate who I knew was also going through some mental health stuff. She said this guy was really good. She actually came to my first appointment.

Therefore, this group of athletes identified facilitators of seeking mental healthcare both within and outside of the sport context. In the case of a number of the athletes, coaches intervened and encouraged mental healthcare when athletes' symptoms manifested in their sport performance or training environment. The nature of the coach-athlete relationship more broadly 
affected how comfortable athletes felt disclosing mental health challenges and seeking support in the sport context. Perceiving the culture of their sport, university, or broader social environments as accepting and supportive of mental health challenges, which was conveyed by exposure to shared experiences, encouraging messaging, and information and visibility of mental healthcare resources, also positively influenced athletes' attitudes toward help-seeking. Finally, other influential individuals were important facilitators of athletes seeking mental healthcare because they identified psychological distress and encouraged the athletes to access professional support, and in some cases facilitated care access more directly by covering the expense of private psychological treatment.

Barriers to Seeking Mental Health Care. While all of the athletes in this group had successfully accessed some degree of mental healthcare prior to participation in the study, they were able to discuss barriers that interfered with their ability to continue accessing care to the extent that they desired, or that delayed them from seeking care.

The coach-athlete relationship. As described in the previous section, on the one hand some of the athletes identified a coach-athlete relationship characterized by acceptance, understanding, and open communication as a powerful facilitator of positive mental health helpseeking attitudes. On the other hand, this important relationship in the lives of competitive athletes clearly also has the potential to interfere with athletes' comfort level in addressing the effects of their mental health on sport performance and seeking support. Salient examples of this barrier are Clara and Mia's experiences:

Clara: My coach had no concept of mental health. I didn’t feel like I had a relationship with him that was productive in any sense in terms of telling him that I was really unhappy or depressed. It's really tough because at the end of the day you're responsible 
for your own happiness but there were definitely times when I just wanted my coach to tell me it's going to be okay, or tell me that maybe I should take today off [training] and that we're going to work at things day by day instead of constantly looking at how we are going to replicate this workout that I dropped out of.

Mia: I never told my coach. I don't know how well he understands it. Just based on his personality I feel like I don't think he would fully get it. So I never told him because I didn't think he would fully get it, or he would be a little confused.

Speaking based on her roles as a coach and athlete, Brenda's perspective sheds light on why Clara and Mia may have experienced a disconnect with their coaches in terms of communicating about mental health concerns. Brenda highlighted a tendency that she has observed in some coaches to focus on the physical when trying to manage performance issues, and thus neglect psychological factors:

Brenda: I think mental health is not something that is attended to enough by coaches or mentors or things like that. We are so focused on, "Oh no they have a stress fracture", "What is their hip mobility?". I think a lot of coaches don't even think about asking, "How are things going?", or "What's going on?". If an athlete is having a bad workout not to assume that it's because they didn't train hard enough, but what else is going on in their life that might make them not be performing well.

While Clara and Mia identified their coaches' lack of mental health literacy and their inability to openly communicate as interfering with their desire to seek support in the sport context, Justin described another type of coach-athlete relationship that acted as a barrier for him to seek support. The dynamic of Justin's relationship with an authoritarian former coach, within which 
emotional expression was discouraged, both contributed to his anxiety and made it feel unacceptable to disclose mental health concerns:

Justin: In macho sport culture you're just supposed to battle through it and find other ways around it as opposed to just opening up about your feelings. Reflecting back on moments from my time in sports where I had incredible anxiety, I think being comfortable to share insecurities with someone would have really helped. It would have done so much for me, but I didn't have a coach who made me feel comfortable to share that with him. I think coaching styles are holding that back.

Therefore, the role of the coach in these athletes' comfort level seeking support for their mental health concerns is complex and depends largely on the nature of the relationship and, in Justin's case, coaching style.

Stigma toward mental illness in sport. Regardless of the presence of a supportive coachathlete relationship, supportive environment, and family and friends encouraging the athletes to seek care, stigma toward mental illness in sport was consistently identified as a significant barrier to athletes seeking treatment. Specifically, the fear of others' perceptions and judgements impacted athletes' desires to admit that were experiencing mental health challenges and therefore their motivation and comfort level to seek treatment.

Heather, Clara, Justin, and Pierre described the fear of being perceived as weak or disadvantaged as an athlete as contributing to denial, and to making them hesitant to engage in help-seeking for mental health treatment:

Heather: As many good things that are going on with Bell Let's Talk and people raising awareness about what's going on, it's such a big stigma in the first place that it's tough to 
come out and say you need help because it's still seen as a disadvantage. I think that's what made it tough for me to talk to my coach about it.

Clara: I would say that a number of athletes struggle with even wanting to admit that they need help. So even if a coach is willing to find those resources for them or connect them with someone or do something, it doesn't necessarily happen because the person is just living in such denial or fear of judgement.

Justin: I think athletes don't know how to talk about it. I think a lot of athletes are totally guilty of hiding their feelings deliberately because of being afraid of being seen as vulnerable.

Pierre: The pressures of teammates and the culture of mental health challenges being devalued, that you're just weak, that was a barrier for sure. I think for a lot of athletes a fallback is that they've pushed through a lot of hard situations, so they're just going to push through this too. They're reluctant to seek help because of that. Instead they just keep pushing on their own, and for stuff like this you often can't manage it by yourself. I wouldn't have gotten through it without the support of other people.

Not only were they afraid of how individuals would perceive them as athletes if they disclosed mental health challenges, Emma and Mia also described being afraid of others' judgements beyond the sport context. It was evident that this fear inhibited their motivation to access treatment and social support:

Emma: If I'm admitted to a program [in-patient or intensive out-patient eating disorder program] I would have had to talk about it and tell people and I wasn't ready or willing to do that. That was a barrier there. As much as I had accepted I had a problem, there is still always an element of denial. I just wasn't ready to admit it to the world. Especially when 
I was younger, I was still in high school and I lived in a small town where everyone talks about everyone. I didn't want it out there. I think I would have gotten better faster if I had talked to people and let them help me, but I also wasn't willing to do that.

Mia: I was always afraid that people would get scared of me because when I was younger I would get scared of people with mental health problems. You don't want to scare people away, especially when you start talking about suicide and stuff like that. It's so overwhelming to other people that they think you're on the edge.

Additionally, for Emma the fear of judgement from others for pursuing mental healthcare was so severe that it caused her to cut her treatment for disordered eating and significant weight loss short after just two counseling sessions:

Emma: The reason I stopped going [to counseling] is because the second time I went I saw someone I knew in the centre and that made me really uncomfortable because part of the reason I went there in the first place was because I didn't want to talk to someone I knew. The person was in my program, not a swimmer, but I didn't want people knowing that I was going. So it wasn't anything about the counseling that was bad, but I didn't want anyone knowing so as soon as I saw someone I knew I was like, "I don't want to go back there and have that happen again".

Financial cost. As highlighted in Table 2, the majority of the athletes in this study accessed mental healthcare through private practice and, not surprisingly, identified the financial cost of such care as a barrier to pursuing the amount of treatment they felt they needed. While the expense of psychological treatment is a barrier for individuals in society in general (Sareen et al., 2007), some athletes highlighted the unique financial burden associated with pursuing competitive sport without the salary or health insurance benefits that come with traditional full- 
time employment, as an additional barrier. For example, Clara described being torn between committing to the multiple counseling sessions she recognized were needed to see improvement in her psychological symptoms and being able to financially support herself in her athletic pursuits:

Clara: I've really struggled largely because it's financially really expensive to see a counselor. You want these immediate results but it's not going to happen because things don't just drastically get better in an hour and a half appointment. The moment I did see a little improvement I would immediately stop seeing whatever counselor I was talking to. Or, on the other hand, I would just be like, “Ugh, this isn't working, it's not worth my time," because I couldn't afford to pay for it while being a starving athlete. We have such little support as it is from a financial standpoint, especially if you're not on the world stage yet, and those resources usually go into physiotherapy and travel to get to competitions.

Brenda reiterated the challenging and unstable financial position that is the reality for many competitive athletes who do not participate in sports rich in endorsements or at a level at which they qualify for government support: "Bottom line I think money is a big barrier. A lot of athletes don't have insurance coverage."

Finally, Raevyn and Mia explicitly stated that the cost of the private psychological treatment that they were able to access prevented them from continuing care, despite believing that it was helpful:

Raevyn: I saw a counselor that was recommended to me by a friend, but it wasn't covered through any health insurance coverage. I only saw her once because it was so 
expensive. It was a useful thing, but it deterred me from going to see her again because of how expensive it was.

Mia: I did feel very guilty about the financial burden because my parents paid for it. I ended my stint of sessions earlier the second time I was depressed and went for treatment because I felt like it was too much money. I stopped going just because of the money. It's so painful but I did pay for probably five sessions, like $\$ 1000$ on my own, but then couldn't afford it so I stopped going even though I felt like...I thought I would be okay but it would obviously be helpful to keep seeing her. But the financial burden was a barrier for sure. I don't even know who could afford that because it's like so much money.

The athletes in this group were evidently drawn to accessing mental health treatment through private practice given that a majority of them pursued this path of care. Even some of the university student-athletes took this route after becoming dissatisfied with the care and length of waitlists for mental health treatment through their university campus health services. While some athletes were able to draw on financial support from their parents, for the majority of study participants the expense of treatment was prohibitive to accessing the amount of care they desired.

\section{Chapter 4: Discussion}

The purpose of this study was to explore the role of competitive sport culture in competitive athletes' experiences with mental illness, the importance athletes place on their mental healthcare provider having an understanding of the sport context, and the avenues by which athletes access mental healthcare to inform barriers and facilitators to care. The aim of the inquiry was to gain an understanding of how the discourses and assumptions inherent to 
normative sport culture affect athlete mental health and to generate knowledge to inform effective psychological treatment of athletes with mental illness. While previous research has explored athletes' perspectives on sport and mental health and mental healthcare (Coyle, Gorczynski, \& Gibson, 2017; Gulliver et al., 2012; Lopez \& Levy, 2013; Watson, 2005), a unique component of this work is the inclusion of competitive athletes who have personal experience with significant mental health challenges and with accessing psychological treatment. The inquiry was informed by social constructionism (Burr, 2015) and a thematic analysis (Braun $\&$ Clarke, 2006) was used to analyze semi-structured interviews with ten athletes. The analysis of these athletes' perspectives was structured into three sections based on the over-arching research questions that informed the study and a description of themes, interwoven with excerpts from the interviews, was presented within each section. Discussion of the implications and conclusions that can be drawn from this work are similarly organized by the topics addressed in each research question.

The first section of the study findings presents a detailed examination of the role of competitive sport culture in both the stressors and benefits athletes perceived as impacting their mental health, and in how athletes have come to perceive mental illness in light of normative discourses that define competitive athletes. Many of the stressors and benefits of sport participation related to athlete mental health that were identified by this group are consistent with previous research (Douglas \& Carless, 2009; Gulliver et al., 2015; Rice et al., 2016). Specifically, the athletes in this study identified experiencing injury or illness that prevented them from training and competing in their sport, performance failures that significantly impacted their sense of self-worth, identity, and sense of control in their lives, and pressure to maintain a particular body shape and physical appearance, as directly contributing to symptoms of 
depression, anxiety, and disordered eating. The athletes also discussed benefits for their mental health derived from competitive sport participation. Often sport represented an important coping mechanism and distraction from negative and anxious rumination, produced perceived physiological benefits, and created opportunities to learn transferable skills related to leadership, time management, and perseverance. This finding is consistent with research that has investigated the development of transferable life skills in the context of youth sport, such as teamwork, goal-setting, time management, emotional skills, interpersonal communication, social skills, leadership, problem-solving, and decision-making (Cronin \& Allen, 2018; Johnston, Harwood, \& Minniti, 2013). Minimal previous work has explored this benefit of sport participation in adult and highly competitive athlete populations, therefore this represents an interesting area for future research. As Reardon and Factor (2010) discuss in their review of the nature of psychopathology in athletes, the relationship between sport and mental illness is complex because of the co-occurrence of negative and positive influences that arise in pursuing sport at a high level. The double-edged sword may be an appropriate metaphor for understanding this relationship in that the athletes described their sport participation as both being responsible for the development of mental illness and for their ability to cope with and overcome psychological distress. These findings shed light on the relevance of the biopsychosocial model of psychopathology etiology in competitive athlete populations (Engel, 1977; DeFreese, 2017). It was evident that the athletes perceived biological, social and psychological mechanisms related to their sport participation to play a role in the development and experience of mental illness. Pierre's perspective in particular highlighted the interaction that likely exists between predisposing characteristics of individuals who are drawn to pursuing competitive sport and the stressors inherent in competitive sport participation for contributing to the development of 
mental health challenges. He believed that components of his personality, such as competitiveness, perfectionism and having single-minded dedication to pursuing goals, selfselected him to being a successful competitive athlete. However, over time these characteristics also made him vulnerable to disordered eating to augment his performance, to developing a strong athletic identity and catastrophic interpretations of performance setbacks, and to the selfstigmatizing attitude that disclosing psychological distress would be perceived as weakness. Interestingly, some research has explored the role of athlete personality traits such as perfectionism, self-esteem, and optimism in the development of eating disorders with equivocal results. For example, personality traits appear to have a small relationship with disordered eating in female athletes but not in male athletes (Galli, Petrie, Greenleaf, Reel, \& Carter, 2014; Petrie, Greenleaf, Reel, \& Carter, 2009) Given these findings and Pierre's experience from the current study, additional research exploring the interplay of pre-disposing characteristics and the sport context on the development of mental illness in athletes is warranted. In addition, considering sport participation as an integral part of the biopsychosocial influences on athlete mental health is recommended for healthcare practitioners who work with this population.

In addressing the first research question an analysis was conducted of how athletes understand mental illness within the context of sport. Findings regarding athletes' perceptions of mental illness in the sport context, represent a significant contribution to athlete mental health literature. Namely, there was an incongruence when the athletes compared their experience with mental illness and the normative discourse of what it means to be a competitive athlete ascribed by their own and societal expectations; that complete dedication and commitment are necessary for success and psychological factors are an unacceptable explanation for performance problems. These findings serve as a potential explanation for the process of self-stigmatization, denial of 
significant psychological distress, and ultimately unfavorable attitudes toward psychological help-seeking that have been consistently reported in this population (Gulliver et al., 2012; Kaier, Cromer, Johnson, Strunk, \& Davis, 2015; Lopez \& Levy, 2013; Watson, 2005). In their examination of the prevalence of mental illness stigma in athletes compared to nonathletes, Kaier and colleagues (2015) found that athletes endorse higher levels of personal and perceived public stigma. Though not specifically explored in this study, the researchers discuss the possibility of aspects of athletic culture contributing to mental illness stigma making treatment seeking feel prohibitive. Based on this suggestion the researchers highlight the need for a research-informed understanding of these cultural dynamics. The findings presented here respond to this suggestion. In summary, based on my analysis I argue that the internal and external expectations to conform to the normative definition of a competitive athlete contributed to athletes who do experience significant mental health challenges perceiving themselves as incongruent with being a mentally tough and capable athlete and that mental illness was an unacceptable excuse for performance issues. Subsequently, these perceptions led to the self-stigmatizing attitude that mental illness is a weakness in the sport domain and seeking support means exposing vulnerability. The normative discourse of what it means to be a competitive athlete described by these athletes was based on their personal expectations as well as their understandings of how the public perceives athletes. Based on this discourse, the competitive athlete is exclusively committed and dedicated to sport performance to the detriment of fostering self-worth and an identity outside of this context and as having impenetrable resilience to the challenges and stressors faced by them and their peers. The existence of this culturally shared belief about competitive athletes is not a new concept in athlete mental health literature. Indeed, it is highly consistent with Douglas and Carless' (2006) concept of the performance narrative, which is a dominant storyline in 
competitive sport culture that exclusive dedication to performance is the only acceptable pursuit for a disciplined and committed elite athlete. In addition, the conclusions that I have drawn regarding the repercussions of the pervasiveness of this discourse in competitive sport culture for athletes' self-stigmatization of mental illness have been discussed, most commonly within critiques of the concept of mental toughness (Bauman, 2016).

The current study contributes to this body of research by producing findings regarding how competitive sport culture produces athletes' perceptions of their mental health challenges as a weakness. This process may be explained using Corrigan and Rao's (2012) theory of the stages of self-stigma. The first stage, “Awareness", occurs when one perceives public stigma about their condition, which the athletes did when they revealed their awareness of public perceptions of competitive athletes. The second stage, "Agreement", occurs when one agrees with negative stereotypes about the group, for example the athletes believing that their psychological symptoms were incongruent with the characteristics of a tough and successful athlete. Finally, "Application" occurs when these stereotypes are applied to the self, which occurred when the athletes described their psychological symptoms as a weakness and representing vulnerability within the sport context. This analysis and interpretation of how self-stigmatization toward mental illness and unfavorable attitudes toward psychological help-seeking develops in athletes embedded in competitive sport culture represents an important theoretical application of this work. In addition, conceptualizing athletes' development of self-stigma in this way informs potential strategies for reducing self-stigma and ultimately making athletes feel more comfortable disclosing mental health concerns.

For instance, an understanding of this process has important implications for how we may change the normative discourse, or create alternative discourses of what it means to be a 
competitive athlete, so that those who do face mental health challenges do not feel that this experience is unacceptable or inconsistent with their and society's view of a successful athlete. One can imagine how a shift in how athletes perceive mental health challenges in the sport context may lead to more favorable attitudes toward help-seeking by considering how physical injuries are addressed. Though extremely challenging for many athletes to cope with, experiencing physical injuries is a common repercussion of competitive sport participation (Appaneal et al., 2009; Clement, Arvinen-Barrow, \& Fetty, 2015; Jacobsson et al., 2013; Yoon, Bae, Kang, \& Kim, 2018). To avoid significant and lengthy interference with sport performance, athletes are encouraged to seek treatment interventions immediately and are even pro-active in managing physical vulnerabilities and minor injuries (Dijkstra, Pollock, Chakraverty, \& Alonso, 2014; Roe et al., 2017). A salient example of this type of response to physical injury is the enforcement of specific protocols for managing concussions by sport governing bodies to facilitate optimal recovery (Ellenbogen et al., 2018). As we learned from the athletes in this study, psychological symptoms significantly interfered with their ability to train and compete optimally in their sports, yet seeking professional treatment for these concerns was often considered as a last resort option because of fear of judgement from individuals in the sport context and personal denial of the severity of the problem.

The findings related to the third research question, which pertain to the barriers and facilitators of athletes' access of mental healthcare, suggest how such a shift in the attitude toward athlete mental health may occur. Namely, the athletes identified features of an accepting environment that demonstrate the type of sport culture that would make them feel more positively toward help-seeking: awareness of shared experiences of other athletes disclosing mental health concerns; receiving encouraging messaging about mental health help-seeking; and 
having access to information about mental health and treatment resources. These findings provide support for Moreland, Coxe, and Yang's (2018) suggestion that supportive attitudes conveyed by stakeholders, such as athletic trainers and administrators, facilitates mental health service utilization. Therefore, a practical recommendation that arises from this work is for sport organizations to take a leading role in implementing these features of an accepting and supportive environment in order to reduce athletes' self-stigma and fear of disclosing mental health challenges.

Attention in some competitive sport organizations has already been drawn to the need for a change in sport culture to prioritizing athlete mental health for the benefit of well-being and performance outcomes in this population. Bauman (2016) highlights this fact in his commentary on efforts by US national and intercollegiate sport governing bodies to emphasize athlete mental health in policy and care recommendations, and it is alluded to in the growing body of research on sport-specific mental health treatment and awareness interventions (Breslin, Shannon, Haughey, Donnelly, \& Leavey, 2017; Gavrilova \& Donohue, 2018). Even the athletes in the current study consistently reported that while they believe considerably more change is needed for athletes to feel comfortable seeking treatment, they do perceive a recent reduction in stigma toward mental illness in athletes. Nonetheless, almost all of them still described the same discourses within competitive sport culture that have been identified as problematic for athlete mental health and well-being (Douglas \& Carless, 2006; Gucciardi, Hanton, \& Fleming, 2017). Therefore, the question arises about what can be done in addition to sport organization- level promotion of athlete mental health awareness and access to support resources to facilitate change in how athletes view their mental health challenges and make them more likely to disclose problematic symptoms. Based on the current findings, a key influencer in athletes' attitudes 
regarding mental health help-seeking is the coach, and the nature of the coach-athlete relationship.

A recent systematic review on mental health symptoms and disorders in elite athletes highlighted a new and growing area of research in this area as examination of the role of coaches as facilitators of access to professional mental health treatment (Castaldelli-Maia et al., 2019). The current findings regarding this group of athletes' perspectives on facilitators of treatment seeking and the coach-athlete relationship lend significant support to the importance of coaches having adequate mental health literacy to be able to identify symptoms and refer athletes to appropriate professional care. Existing research in this area typically includes samples of athletes who do not necessarily have personal experience accessing mental healthcare (Biggin, Burns, \& Uphill, 2017; Delenardo \& Terrion, 2014; Kroshus, 2017; Wahto, Swift, \& Whipple, 2016). Though these participants' perspectives undoubtedly shed light on the potential role that coaches may have as barriers and facilitators of mental health help-seeking, the current study extends this area of research by accessing the perspectives of athletes with lived experience in this domain.

This unique perspective revealed that identifying psychological symptoms, directly recommending that athletes seek treatment, and fostering a relationship characterized by acceptance, understanding, and open communication about sport and non-sport related stressors are the specific ways in which coaches acted as help-seeking facilitators. Interestingly, these elements of the coach's role are highly consistent with the views of coaches interviewed by Mazzer and Rickwood (2015) about their role in supporting the mental health of their adolescent athletes. These coaches believed that being approachable and communicating about mental health concerns and identifying signs of psychological distress were inherent to their responsibility of promoting the well-being of the athletes. The coaches in Mazzer and 
Rickwood's (2015) study reported that actually helping to treat athletes' mental disorder symptoms is beyond the scope of their role, which is a critical distinction to make when suggesting that coaches be actively involved in facilitating athlete mental healthcare. Therefore, based on the current findings, which are consistent with previous research that has explored both athletes' and coaches' views on the role of the coach in influencing attitudes toward mental health help-seeking, it is recommended that sport organizations or coaching accreditation systems mandate mental health literacy training for competitive sport coaches so that they may identify signs of mental ill-health and be aware of local and accessible avenues through which to refer athletes to appropriate professional care. Based on a recent study in Australia, brief mental health literacy training for elite sport staff is effective in increasing coaches' knowledge and confidence in helping vulnerable athletes (Sebbens, Hassmen, Crisp, \& Wensley, 2018). Coaches, trainers and support staff working with Australian competitive athlete underwent a four-hour workshop designed to educate these individuals to recognize, reach out, refer to appropriate care and provide social support to athletes experiencing mental health challenges. Post-intervention the participants reported significant improvement in depression and anxiety literacy and self-reported confidence in their ability to help someone experiencing a mental health problem (Sebbens et al., 2018). These encouraging preliminary findings indicate that future research should explore the implementation and dissemination of such training across competitive sport contexts.

Thus far conclusions and implications related to my first and third research questions have been discussed, which referred to the relationship between sport culture and athlete mental health and barriers and facilitators to mental health care. These two areas were evidently highly related, with self-stigma produced by a normative discourse ascribing to competitive athletes 
superhuman psychological characteristics representing a powerful barrier to mental health helpseeking, and an accepting sport environment and coaches acting as critical facilitators. The final research question, for which conclusions and implications must be explored, is athletes' perspectives on the importance of their mental healthcare providers having specific knowledge of or experience with the sport context.

As with most areas of athlete mental heath research, growing attention has been paid to the concept of sport-specific mental health interventions and the necessity of specialized mental healthcare providers to work with athlete populations (Gavrilova \& Donohue, 2018; Moesch et al., 2018). Out of this area of work, and of particular relevance to the current study, a consistent recommendation is made that mental healthcare providers who treat athletes must have knowledge of the sport context to be able to provide optimal treatment. For example, based on their review of models of mental health service provision in six European countries Moesch and colleagues (2018) reported that "all authors agree that the treatment of clinical disorders in elite sports should be conducted by persons who have a thorough knowledge of the nature of competitive contexts" (Moesch et al., 2018, p. 68). The authors elaborate that this type of knowledge may be acquired through qualification as a sport psychologist, other specific education in sport science and competitive contexts, or personal experience in elite sport or with working with athletes. There is an intuitive logic to this assertion that has led many researchers to recommend such qualifications for working with athletes, especially considering the growing body of evidence from the current study and others that athletes' mental health challenges and even symptoms of clinical mental disorders may be related to participation in competitive sport (Reardon et al., 2019). However, there is a need to critically examine this recommendation as much of the research it is based on has investigated athletes' perspectives who have not actually 
had personal experience working with a mental healthcare provider (Donohue et al., 2004; Lopez \& Levy, 2013; Watson, 2005). Herein lies the unique contribution of the current study, and by exploring competitive athletes' perspectives who have personal experience to reflect on, a much more nuanced understanding of this question is revealed.

The perspective shared by this group of athletes was that sport specific knowledge or experience could indeed be a significant asset in their mental healthcare providers and was certainly highly valued by the few athletes who actually accessed care providers with this expertise. A conclusion based on the analysis of these athletes' experiences that has not been explored in previous research is that the degree to which the athletes perceived their mental health to be intertwined with their sport participation was related to how important they believed it was for their care provider to be familiar with this context. The reality was, however, that based on the experiences of this group of Canadian competitive athletes, accessing clinical psychologists, psychiatrists, or counsellors with expertise in the sport domain was rare. This is not entirely surprising given that there are no education opportunities that specifically train mental healthcare providers in athlete mental health treatment in Canada, and the first centre offering specialized mental healthcare for competitive athletes and coaches opened only recently in Canada in 2018 (Van Slingerland et al., 2019). Therefore, while access to this kind of specialized care may improve in the near future, many competitive athletes will continue to receive treatment from their local mental healthcare providers, who, as we have learned from the current study are likely not to have sport-specific knowledge. Thus, these athletes' discussion of components of successful treatment experiences, despite their provider not having sport-specific expertise, is informative. This perspective revealed that validating the role that sport played in the athletes' lives and making a concerted effort to understand the psychological and lifestyle 
demands that a competitive athlete experiences was often sufficient for rapport building. The pairing of good rapport and the mental healthcare provider demonstrating intelligence and professional expertise was satisfactory for many of the athletes interviewed for this study. This finding is reassuring given that requiring mental healthcare providers working with athletes to have sport-specific knowledge may act as an additional barrier to accessing treatment by limiting the number of qualified providers. It also has important implications for mental healthcare providers without extensive prior experience working with competitive athletes who find themselves exposed to this population Accessing research such as that presented in this study exploring the elements of competitive sport culture that athletes perceive as playing a role in their mental health can serve as a valuable resource for these mental healthcare providers.

An additional recommendation relevant to mental healthcare providers is the importance of considering the sport context when assessing mental disorders in athletes. This consideration should be made when assessing the development and nature of specific symptoms as well as the functional impact of the mental disorder on an athlete's life. As demonstrated in the current study and discussed in detail in Reardon and colleagues' (2019) review, the etiology of certain mental health issues such as mood, anxiety, and eating disorders may be related to the cultures of some competitive sports. In addition, specific symptoms may have atypical manifestations in the sport context, and sport participation may also be an important coping mechanism for athletes experiencing mental health challenges (Reardon et al., 2019). Furthermore, it is clear that the ability to train and perform in their respective sports is often highly intertwined with competitive athletes' sense of self-worth and personal identity, which is associated with negative mental health repercussions when injury or illness interfere with sport performance. Therefore, in terms of assessing for the extent of functional impact and clinical distress associated with symptoms, 
which is included as a diagnostic criteria for almost all disorders in the Diagnostic and Statistical Manual $5^{\text {th }}$ Edition (DSM-5) (American Psychiatric Association, 2013), the domain of sport should be considered alongside social, occupational, and educational functioning.

\section{Conclusions and Future Directions}

Implications and applied recommendations based on the current study have been highlighted throughout this discussion and they will be summarized here, along with strengths and limitations of the study design, implementation, and analysis and suggestions for future research. In terms of the relationship between competitive sport culture and athlete mental health, a theoretical process was presented based on Corrigan and Rao's (2012) stages of selfstigma to explain how the incongruence between the culturally produced normative discourse of being a competitive athlete and the reality of these athletes' experiences with mental illness contributed to athletes' self-stigmatizing attitude of psychological symptoms being a weakness and vulnerability. From this perspective, shifting the discourse around mental illness and sport to be more in line with that surrounding physical injury and illness, which are managed through preventative, embedded, and often easily accessible resources, will likely reduce self-stigma and improve athletes' attitudes toward help-seeking. Such a shift may occur through athletes sharing their experiences with mental health challenges, sport organizations distributing encouraging messaging and information about mental health and treatment resources, and through coaches developing adequate mental health literacy to identify and appropriately communicate with vulnerable athletes and facilitate access to professional treatment. Furthermore, implications of the analysis of the athletes' perspectives on their mental healthcare providers having specific knowledge of the sport context and recommendations for care providers were discussed. Care providers with this expertise were valued by the athletes because of their ability to normalize, 
validate, and understand the sport-specific dimensions of their mental health experiences based on prior exposure to athletes with similar experiences. An informative takeaway from the athletes' perspectives, however, is that psychological treatment could still be beneficial if the care provider did not have specific knowledge of the competitive sport context if sufficient rapport was established through valuing and engaging with the athlete's sport experience. Finally, an important applied recommendation is for the consideration of the relationship between the sport context and mental disorder symptoms throughout assessment and treatment when working with an athlete who perceives their mental health to be intertwined with their sport participation.

There are strengths and limitations of the study design, implementation, and analysis that must be acknowledged to contextualize the contributions of this research. A significant strength lies in the participant sample because of their personal experience with mental healthcare, experiences with a range of individual and team sport cultures, and range of ages and types of sport involvement from being former competitive athletes with the ability to reflect on changes in sport culture since their involvement to current, full-time competitive athletes. Anchored in the social constructionist paradigm that informed the current study, the analysis of these athletes' perspectives cannot necessarily be assumed to generalize to all competitive athletes with mental health challenges. That being said, there are likely similarities between these athletes' experiences and those who share elements of the social, historical, and cultural contexts in which these experiences are embedded. An additional strength is the rich detail with which the athletes shared their experiences and how this detail is highlighted throughout the study results by the incorporation of long excerpts from the interviews. In keeping with the subjectivist epistemology underpinning my thematic analysis, according to which knowledge generation is considered a 
joint production between the researcher and participation (Guba, 1996), it was important to not only incorporate but emphasize the participants' voices in the research product.

Finally, highlighting limitations of the current study allows us to consider fruitful future avenues of inquiry that relate to the findings presented here. As mentioned above, the participant sample varied in some characteristics, while gender and race were less variable with the majority of the athletes interviewed being female and white. The homogeneity of the sample with respect to these characteristics is worth considering given that cultural dynamics related to gender and race impact athlete mental health and attitudes toward mental health help-seeking (CastaldelliMaia et al., 2019). While exploring the potentially gendered nature of competitive athlete mental health experiences was not a focus of the current study, one female athlete and both of the male athletes who participated spontaneously mentioned perceived effects of gender on their understanding of mental health in the sport context. Research has recently begun exploring gender differences in mental health help-seeking attitudes and behaviors in athletes, with inconsistent results suggesting that the male gender may be associated with higher levels of selfstigma toward mental disorders and help-seeking (Barnard, 2016; Hilliard, Redmond, \& Watson, 2018; Jones, 2016). This inconsistency indicates that additional research in this area is needed to shed light on whether different barriers and facilitators to care are experienced related to gender. In addition, based on the perspectives shared by the three athletes mentioned here and previous research that has specifically examined male athletes' experiences with mental illness, there may be different cultural elements in male versus female competitive sport that influence athletes' experiences of mental illness. Such elements include "macho" or hypermasculine expectations to suppress emotions and maintain a façade of aggression and strength in male culture (Doherty, Hannigan, \& Campbell, 2016; Souter, Lewis, \& Serrant, 2018). More research is needed to 
explore this area and specifically the gendered experience of female athletes which has received comparatively little research attention (Jones, Butryn, Furst, \& Semerjian, 2013).

Other areas that would benefit from additional research are in athlete mental healthcare and mental health literacy for coaches and other athlete support personnel. To continue to develop our understanding of competitive athletes' experiences with mental healthcare and generate knowledge to enhance access to effective and appropriate treatment, the perspectives of mental healthcare providers who have had experience working with athletes should be explored. It would be informative to compare their perspectives on the importance of having specific knowledge of the sport context when working with athletes to the views expressed by the athletes in the current study. In terms of enhancing mental health literacy in support personnel, and more generally creating cultures in sport organizations that encourage mental health helpseeking in athletes, the existing practices of Canadian sport organizations to support the mental health of athletes should be investigated and dissemination of awareness and training interventions be implemented.

In summary, the aim of the current study was to explore, in-depth, competitive athletes' perspectives on the relationship between their sport participation, including the role of competitive sport culture, and their mental health, as well as their experiences with mental healthcare. The analysis of this group of ten competitive athletes' experiences represents a unique contribution to the burgeoning field of athlete mental health research by sharing the perspectives of athletes who were specifically recruited to participate based on their personal experience with clinically significant mental health concerns and with accessing mental healthcare. This approach allowed for the opportunity to critically examine previous conclusions drawn based on research with general athlete participants in the following areas: the role of 
competitive sport culture and self-stigma on attitudes toward mental health help-seeking; barriers and facilitators to accessing mental healthcare; and the importance of a mental healthcare provider having expertise in the sport domain or the effective treatment of athletes. 


\section{Interview Guide}

\section{Appendix A}

1. Can you tell me about yourself?

a. Prompts: how old are you? What is your occupation? Where are you from?

2. Are you currently involved in sport? If so, can you explain your involvement? If not, when were you last involved in sport?

a. Prompts: what sport(s) do you participate in? How would you describe the level of your sport participation?

3. How would you describe the highest level of sport you have participated at?

4. What has your sport experience been like?

a. Prompts: what is the value of sport to you? Do you have particularly positive or negative views of sport? Why do you participate in sport?

5. How would you describe competitive sport culture?

6. What do you think about mental health in sport?

a. Prompts: do you think sport is related to mental health, why or why not?

7. How do you think athletes view mental health? Coaches? Society in general?

8. What is your experience with mental health challenges?

9. What are your thoughts about the relationship between your sport participation and your mental health?

a. Prompts: were the reasons you sought mental healthcare related to your experience as an athlete?

10. What kind of experiences have you had with mental healthcare? 
a. Prompts: Did you seek/receive treatment? What type of clinician did you see? Who suggested you seek treatment? Who referred you to treatment? What kind of treatment did you receive? Where did you receive treatment?

11. Was the clinician you received treatment from experienced with sport or athletes' experiences?

12. Do you think a clinician having in-depth knowledge of sport could affect athletes' treatment experiences?

13. What do you think is most important within the therapist-patient relationship for effective treatment?

14. Are there any barriers to mental healthcare that athletes face?

15. Is there anything else you want to add or highlight? 


\section{Appendix B}

\section{Mental Health Resources}

\section{Information from Canadian Mental Health Association Toronto Find Help}

\section{Getting Help: When and How}

Most of us go through life solving our day-to-day problems without needing help to cope with our feelings. But sometimes, things get out of hand. A severe illness, an accident or an emotional crisis can overwhelm us, at least temporarily, and suddenly we need help.

\section{How do you know if you need help?}

Sometimes the need for help is obvious, and getting it is as simple as phoning for an ambulance or a fire truck. At other times, it can be hard to admit help is needed. This is especially true when your emotions are involved. The problem may be anything from what to do about an aging and increasingly helpless parent to a serious emotional problem such as depression. Here are some of the reasons you may decide you need help:

- You find yourself feeling overwhelmed by feelings of anger or despair, and you cannot enjoy life anymore.

- You used to be healthy, but now you are always feeling a bit sick and you are missing more and more time from work.

- Your finances are out of control, and you are worried about being able to pay the next month's rent or mortgage payment.

- You cannot "get over" the death of someone you loved very much.

- There is too much conflict at home. You are afraid your marriage may break up.

- You are drinking too much or having some other kind of drug problem.

- You are feeling suicidal.

\section{What kind of help is available?}

There are many different kinds of assistance available, and you should be able to find the help you need within your community through the following sources:

Psychiatrist

Your family doctor may refer you to a psychiatrist who is a medical doctor specially trained in the diagnosis and treatment of mental illnesses. He/she may treat your problems with medication or by psychotherapy (sometimes called "talk therapy"), or a combination of both. You can search for doctors who are accepting new patients on the College of Physicians and Surgeon's website: www.cpso.on.ca

Psychologist

You may decide to seek help from a psychologist, and you do not need a referral from your family doctor 
to do so. A psychologist will have a doctoral degree from a university but not a medical degree. He/she will use counselling and other methods that do not involve the use of medications. If you plan to see a psychologist, you should remember that his/her services are not necessarily fully covered by public health insurance. You may want to find out if some coverage is available through private insurance (for example, your company benefits plan) or through social assistance. You can find a psychologist by contacting the Ontario Psychological Association: 416-961-5552.

Other Therapist

Your family doctor or a psychiatrist may refer you to a therapist such as a social worker with specialized training. Again, you should be aware that the services offered may not be covered by an insurance plan. To speak with an Information Referral Specialist about what options are available to you contact Connex Ontario. The Mental Health Helpline provides information about mental health services in Ontario: 1-866-531-2600 or search their web directory: http://www.mentalhealthhelpline.ca/

You can also Click Here to access a Psychotherapy Referral List published by Making The Links at the Hassle Free Clinic. (http://toronto.cmha.ca/files/2012/08/Psychotherapist-LIST july2012-pdf.pdf)

\section{Self-Help Group}

You may find it helpful to join a self-help group. These groups provide the mutual support of people who have all had similar experiences. For example, there are groups for people suffering from depression, grief, the trauma of sexual assault, eating disorders, and phobias (a phobia is an irrational, crippling fear of an object, animal or situation). To find a local self-help group that can meet your needs contact the Self-Help Resource Centre Info Line: 416-487-4355 or search their web directory: http://www.selfhelp.on.ca/

\section{Other Community Services}

You may find that some of your problems can be solved by assistance from agencies outside the mental health system. Sometimes, practical help, such as home nursing care, Meals On Wheels or subsidized door-to-door transportation for people unable to walk, will greatly reduce the stress in your life, either as a care-giver or as a disabled person. To find what local services may be available to you can contact Toronto's Find Help Information Services by dialing: 211 or searching their web directory: http://www.211toronto.ca/index.jsp

Help from Friends and Others

Sometimes, the help of a trusted family member, a close friend or a member of the clergy for your religion can be a source of support. People close to you can also point you in the direction of the help you need. 


\section{Are You in Crisis?}

If you are in a crisis and require emergency assistance:

-Visit your local emergency department or call 911

-Contact one of the Crisis Response Programs serving Toronto below:

Anishnawbe 24/7 Mental Health Crisis Management Service: 416-891-8606 (Aboriginal clients) not only promotes Traditional Aboriginal practices but has affirmed and placed them at its core. Its model of health care is based on Traditional practices and approaches and are reflected in the design of its programs and services.

Assaulted Women's Helpline: 416-863-0511; Toll Free 1-866-863-0511 For more than 25 years, the Assaulted Women's Helpline has served as a free, anonymous and confidential 24hour telephone and TTY crisis telephone line to all women in the province of Ontario who have experienced any form of abuse.

Distress Centre: 416-408-HELP (4357) offers access to emotional support from the safety and security of the closest telephone. Callers can express their thoughts and feelings in confidence. Callers' issues can include problems related to domestic violence, social isolation, suicide, addictions, mental and physical health concerns. The Distress Centre offers emotional support, crisis intervention, suicide prevention and linkage to emergency help when necessary.

Gerstein Centre: (416) 929-5200 provides crisis intervention to adults, living in the City of Toronto, who experience mental health problems. The service has three aspects; telephone support, community visits and a ten-bed, short-stay residence. All three aspects of the service are accessed through the crisis line.

Kids Help Phone: 1-800-668-6868 is a free, anonymous and confidential phone and on-line professional counselling service for youth. Big or small concerns. 24/7. 365 days a year.

York Support Services Network: 1-855-310-COPE (2673) 1-866-323-7785 (TTY) If you, or someone you care about, are depressed, distressed, lonely, anxious, scared or angry, you can call York Support Services 24/7. They have been serving York Region for over 15 years and have now expanded their telephone support to include North York.

Scarborough Hospital Mobile Crisis Program: 416-495-2891 provides Telephone Crisis Response and community crisis response to individuals over 16 years of age who are experiencing a mental health crisis inScarborough and East York.

Senior Crisis Access Line: 416.619.5001 is a specialized service for seniors in the Toronto Central LHIN areawhich provides assistance in stabilizing age-related mental health and 
addiction crises, risk and safety assessments, immediate referrals to community mobile crisis units, and support for families and caregivers.

Toronto Rape Crisis Centre: $\mathbf{4 1 6 - 5 9 7 - 8 8 0 8}$ is a grassroots collective working towards a violence-free world by providing anti-oppressive, feminist peer support to survivors of sexual violence through support, education and activism. Callers can be anyone who has been raped, sexually assaulted or abused, women who have had unwanted sexual touching, incest survivors and friends or family.

Youthdale's Crisis Support Team: (416) 363-9990 assesses each child's risk and mental status through telephone interviews and if required, Mobile Response. This assessment will involve the parent or guardian and the professionals caring for the child. A Youthdale child psychiatrist is available to consult with the Crisis Support Team at all times. Any parent or legal guardian in Ontario can call the Youthdale Psychiatric Crisis Services and get immediate access to trained professionals 24 hours a day, seven days a week.

\section{Emergency Shelter}

Central Intake: 416-338-4766; Toll Free 1-877-338-3398

Streets to Homes Assessment and Referral Centre (129 Peter Street): 416-392-0090 Walk in referral to emergency shelter and street respite.

\section{If you are looking for Information \& Referral services in Ontario:}

ConnexOntario offers province-wide information and referral services for those with mental health or addiction challenges. You can visit their website at: web address, or you can call the following numbers which operate 24 hours a day, 7 days a week:

- $\quad$ Mental Health Helpline, 1-866-531-2600

- Drug and Alcohol Helpline, 1-800-565-8603

- $\quad$ Ontario Problem Gambling Helpline, 1-888-230-3505 
Recruitment Poster

Appendix C

\section{COMPETITIVE ATHLETES' EXPERIENCES WITH MENTAL ILLNESS AND PSYCHOLOGICAL TREATMENT}

ARE YOU:

- 18 years or older?

- Male, female, other?

- A current or former competitive athlete?

DO YOU HAVE:

- Experience with psychiatric or psychological treatment for mental health challenges?

If you can answer yes to all of the questions above, please consider participating in a research interview.

THE INTERVIEW:

- 45-60 minutes

- $100 \%$ confidential

- Asks about your experience with mental health as an athlete, accessing mental healthcare, and your perceptions of your treatment experience 


\section{References}

Ackerman, S. J., \& Hilsenroth, M. J. (2003). A review of therapist characteristics and techniques positively impacting the therapeutic alliance. Clinical Psychology Review, 23, 1-33.

American Psychiatric Association (2013). Diagnostic and statistical manual of mental disorders (5th edition). Washington, DC: American Psychiatric Association.

Andersen, M. B., Denson, E. L., Brewer, B. W., \& Van Raalte, J. L. (1994). Disorders of personality and mood in athletes: Recognition and referral. Journal of Applied Sport Psychology, 6, 168-184.

Anderson, A., Miles, A., Robinson, P., \& Mahoney, C. (2004). Evaluating the athlete's perception of the sport psychologist's effectiveness: What should we be assessing? Psychology of Sport and Exercise, 5, 255-277.

Appaneal, R. N., Levine, B. R., Perna, F. M., \& Rohl, J. L. (2009). Measuring postinjury depression among male and female competitive athletes. Journal of Sport and Exercise Psychology, 31, 60-76.

Araújo, C. G. S., Scharhag, J. (2016). Athlete: A working definition for medical and health sciences research. Scandinavian Journal of Medicine and Science in Sports, 26, 4-7.

Barnard, J. D. (2016). Student-athletes' perceptions of mental illness and attitudes toward helpseeking. Journal of College Student Psychotherapy, 30, 161-175.

Bauman, J. N. (2016). The stigma of mental health in athletes: Are mental toughness and mental health seen as contradictory in elite sport? British Journal of Sports Medicine, 50, 135136. 
Biggin, I., J., R., Burns, J. H., \& Uphill, M. (2017). An investigation of athletes' and coaches’ perceptions of mental ill-health in elite athletes. Journal of Clinical Sport Psychology, 11(2), 126-147.

Bordin, E. S. (1976). The generalizability of the psychoanalytic concept of the working alliance. Psychotherapy: Theory, Research and Practice, 16, 252-260.

Braun, V., \& Clarke, V. (2006). Using thematic analysis in psychology. Qualitative Research in Psychology, 3, 77-101.

Breslin, G., Shannon, S., Haughey, T., Donnelly, P., \& Leavey, G. (2017). A systematic review of interventions to increase awareness of mental health and well-being in athletes, coaches, and officials. Systematic Reviews, 6(177), 1-15.

Brewer, B. W., Van Raalte, J. L., \& Linder, D. E. (1993). Athletic identity: Hercules' muscles or Achilles' heel? International Journal of Sport Psychology, 24, 237-254.

Broughton, E., \& Neyer, M. (2001). Advising and counseling student athletes. New Directions for Student Services, 93, 47-53.

Burr, V. (2015). Social constructionism ( ${ }^{\text {rd }}$ ed.). New York, NY: Routledge.

Castaldelli-Maia, J., Gallinaro, J. G. M., Falcão, R. S., Gouttebarge, V., Hitchcock, M. E., Hainline, B., ... Stull, T. (2019). Mental health symptoms and disorders in elite athletes: A systematic review on cultural influencers and barriers to athletes seeking treatment. British Journal of Sports Medicine, 53, 707-721.

Chamberlain, K. (2000). Methodolatry and qualitative health research. Journal of Health Psychology, 5, 285-296. 
Clement, D., Arvinen-Barrow, M., \& Fetty, T. (2015). Psychosocial responses during different phases of sport-injury rehabilitation: A qualitative study. Journal of Athletic Training, 50, 95-104.

Corrigan, P. W., \& Rao, D. (2012). On the self-stigma of mental illness: Stages, disclosure, and strategies for change. The Canadian Journal of Psychiatry, 57(8), 464-469.

Coyle, M., Gorczynski, P., \& Gibson, K. (2017). "You have to be mental to jump off a board anyway": Elite divers' conceptualizations and perceptions of mental health. Psychology of Sport and Exercise, 29, 10-18.

Creswell, J. W. (2007). Qualitative inquiry and research design: Choosing among five approaches. Thousand Oaks, CA: Sage.

Cronin, L. D., \& Allen, J. (2017). Development and initial validation of the Life Skills Scale for Sport. Psychology of Sport and Exercise, 28, 105-119.

Daly, K. J. (2007). Qualitative methods for family studies and human development. Thousand Oaks, CA: Sage.

DeFreese, J. D. (2017). Athlete mental health care within the biopsychosocial model. Athletic Training and Sports Health Care, 9(6), 243-245.

Delenardo, S., \& Terrion, J. L. (2014). Suck it up: Opinions and attitudes about mental illness stigma and help-seeking behavior of male varsity football players. Canadian Journal of Community Mental Health, 33(3), 43-56.

DeSantis, L., \& Noel Ugarriza, D. (2000). The concept of theme as used in qualitative nursing research. Western Journal of Nursing Research, 22, 351-372. 
Dijkstra, H. P., Pollock, N., Chakraverty, R., \& Alonso, J. M. (2014). Managing the health of the elite athlete: A new integrated performance health management and coaching model. British Journal of Sports Medicine, 48, 523-531.

Doherty, S., Hannigan, B., \& Campbell, M. J. (2016). The experience of depression during the careers of elite male athletes. Frontiers in Psychology, 7(1069), 1-11.

Donohue, B., Dickens, Y., Lancer, K., Covassin, T., Hash, A., Miller, A., \& Genet, J. (2004). Improving athletes' perspectives of sport psychology consultation: A controlled evaluation of two interview methods. Behaviour Modification, 28(2), 182-193.

Douglas, K. \& Carless, D. (2009). Abandoning the performance narrative: Two women's stories of transition from professional sport. Journal of Applied Sport Psychology, 21, 213-230.

Douglas, K. \& Carless, D. (2015). Life story research in sport: understanding the experiences of elite and professional athletes through narrative. Abingdon, New York: Routledge.

Douglas, K., \& Carless, D. (2006). Performance, discovery, and relational narratives among women professional tournament golfers. Women in Sport and Physical Activity Journal, $15,14-27$.

Ellenbogen, R. G., Batjer, H., Cardenas, J., Berger, M., Bailes, J., Pieroth., ...Sills, A. (2018). National Football League head, neck and spine committee's concussion diagnosis and management protocol: 2017-2018 season. British Journal of Sports Medicine, 52(14), 894-902.

Ellis, C. Kiesinger, C. E., \& Tillmann-Healey, L. (1997). Interactive interviewing: Talking about emotional experience. In R. Herz (Ed.), Reflexivity and voice (pp. 119-149). Newbury Park, CA: Sage. 
Engel, G. (1977). The need for a new medical model: A challenge for biomedicine. Science, 196, 129-136.

Fontana, A. \& Frey, J. H. (2000). Interviewing: The art of science. In N. K. Denzin \& Y. S. Lincoln (Eds.), Collecting and interpreting qualitative materials (pp. 47-78). Newbury Park, CA: Sage.

Fourie, S., \& Potgieter, J. R. (2001). The nature of mental toughness in sport. South African Journal for Research in Sport, Physical Education and Recreation, 23, 63-72.

Galli, N., Petrie, T. A., Greenleaf, C, Reel, J. J., \& Carter J. E. (2014). Personality and psychological correlates of eating disorder symptoms among male collegiate athletes. Eating Behaviors, 15(4), 615-618.

Gavrilova, Y., \& Donohue, B. (2018). Sport-specific mental health interventions in athletes: A call for optimization models sensitive to sport culture. Journal of Sport Behavior, 41(3), 283-304.

Glick, I. D., \& Horsfall, J. L. (2009). Psychiatric conditions in sports: Diagnosis, treatment, and quality of life. The Physician and Sportsmedicine, 37, 29-34.

Glick, I. D., Stillman, M.A., Reardon, C.L., \& Ritvo, E. (2012). Managing psychiatric issues in elite athletes. Journal of Clinical Psychiatry, 73, 640-644.

Gorczynski, P. F., Coyle, M., \& Gibson, K. (2017). Depressive symptoms in high-performance athletes and non-athletes: A comparative meta-analysis. British Journal of Sports Medicine, 51, 1348-1354.

Green, G. A., Uryasz, F. D., Petr, T. A., \& Bray, C. D. (2001). NCAA Study of Substance Use and Abuse Habits of College Student-Athletes. Clinical Journal of Sport Medicine, 11, 51-56. 
Guba, E. G. \& Lincoln, Y. S. (1994). Competing paradigms in qualitative research. In N. K. Denzin \& Y. S. Lincoln (Eds.), Handbook of qualitative research (pp. 105-117). Thousand Oaks, CA: Sage.

Guba, E. G. (1996). What happened to me on the road to Damascus. In L. Heshusius \& K. Ballard (Eds.), From positivism to interpretivism and beyond: Tales of transformation in educational and social research (pp. 43-49). New York: Teachers College Press.

Gucciardi, D. F., Hanton, S., \& Fleming, S. (2017). Are mental toughness and mental health contradictory concepts in elite sport? A narrative review of theory and evidence. Journal of Science and Medicine in Sport, 20, 307-311.

Gulliver, A., Griffiths, K. M., \& Christensen, H. (2012). Barriers and facilitators to mental health help-seeking for young elite athletes: A qualitative study. BMC Psychiatry, 12, 157-171.

Gulliver, A., Griffiths, K. M., Mackinnon, A., Batterham, P. J., \& Stanimirovic, R. (2015). The mental health of Australian elite athletes. Journal of science and medicine in sport, 18, 255-261.

Hilliard, R. C., Redmond, L. A., \& Watson, J. C. (2018). Differences in stigma and attitudes toward counseling between college student-athletes and nonathletes. Journal of College Student Psychotherapy, 11(3), 1-8.

Jacobsson, J., Timpka, T., Kowalski, J., Nilsson, S., Ekberg J., Dahlstrom, O., \& Renstrom, P. A. (2013). Injury patterns in Swedish elite athletics: Annual incidence, injury types, and risk factors. British Journal of Sports Medicine, 47, 941-952.

Jayasekara, R., Procter, N., Harrison, J., Skelton, K., Hampel, S., Draper, R., \& Deuter, K. (2015). Cognitive behavioural therapy for older adults with depression: A review. Journal of Mental Health, 24, 168-171. 
Jewett, R., Kerr, G., \& Tamminen, K. (2019). University sport retirement and athlete mental health: A narrative analysis. Qualitative Research in Sport, Exercise and Health, 11(3), 416-433.

Jewett, R., Sabiston, C. M., Brunet, J., O’Loughlin, Scarapicchia, T., \& O’Loughlin, J. (2014). School sport participation during adolescence and mental health in early adulthood. Journal of Adolescent Health, 55, 640-644.

Joe, G. W., Stimpson, D. D., Dansereau, D. F., \& Rowan-Szai, G. A. (2001). Relationships between counseling rapport and drug abuse treatment outcomes. Psychiatric Services, 52, 1223-1229.

Johnston, J., Harwood, C., \& Minniti, A. M. (2013). Positive youth development in swimming: Clarification and consensus of key psychosocial assets. Journal of Applied Sport Psychology, 25(4), 392-411.

Jones, A. L., Butryn, T. M., Furst, D. M., \& Semerjian, T. Z. (2013). Phenomenological examination of depression in female collegiate athletes. Athletic Insight, 5, 1-19.

Jones, G., Hanton, S., \& Connaughton, D. (2002). What is this thing called mental toughness? An investigation of elite sport performance. Journal of Applied Sport Psychology, 14, 205-218.

Jones, T. V. (2016). Predictors of perceptions of mental illness and averseness to help: A survey of elite football players. Journal of Mental Health, 25, 422-427.

Kaier, E., Cromer, L. D., Johnson, M. D., Strunk, K., \& Davis, J. L. (2015). Perceptions of mental illness stigma: Comparisons of athletes to nonathlete peers. Journal of College Student Development, 56(7), 735-739.

Kerr, G. and Dacyshyn, A., 2000. The retirement experiences of elite, female gymnasts. Journal of applied sport psychology, 12 (2), 115-133. 
Kroshus, E. (2017). Stigma, coping skills, and psychological help-seeking among collegiate athletes. Athletic Training and Sports Health Care, 9(6), 254-262.

Lambert, M. J., \& Barley, D. E. (2001). Research summary on the therapeutic relationship and psychotherapy outcome. Psychotherapy, 38, 357-361.

Lavallee, D. \& Robinson, H. K. (2007). In pursuit of an identity: A qualitative exploration of retirement from women's artistic gymnastics. Psychology of Sport and Exercise, 8, 119141.

Lavallee, D., Gordon, S., \& Grove, J. R. (1997). Retirement from sport and the loss of athletic identity. Journal of Personal and Interpersonal Loss, 2, 129-147.

Lopez, R. L., \& Levy, J. J. (2013). Student athletes' perceived barriers to and preferences for seeking counseling. Journal of College Counseling, 16, 19-31.

Lubker, J. R., Visek, A. J., Geer, J. R., \& Watson, J. C. (2008). Characteristics of an effective sport psychology consultant: Perspectives from athletes and consultants. Journal of Sport Behavior, 31, 147-165.

MacKenzie, K. R. \& Tschuschke, V. (1993). Relatedness, group work, and outcome in long-term inpatient psychotherapy groups. Journal of Psychotherapy Practice and Research, 2, 147-156.

Maniar, S. D., Curry, L. A., Sommers-Flanagan, J., \& Walsh, J. A. (2001). Student-athlete preferences in seeking help when confronted with sport performance problems. The Sport Psychologist, 15, 25-223.

Mann, B. J., Grana, W. A., Indellcato, P. A., O’Neill, D. F., George, S. Z. (2007). A survey of sports medicine physicians regarding psychological issues in patient-athletes. The American Journal of Sports Medicine, 35, 2140-2147. 
Martin, S. B., Akers, A., Jackson, A. W., Wrisberg, C. A., Nelson, L., Leslie, P. J., \& Leidig, L. (2001). Male and female athletes' and nonathletes' expectations about sport psychology consulting. Journal of Applied Sport Psychology, 13, 18-39.

Mazzer, K. R., \& Rickwood, D. J. (2015). Mental health in sport: Coaches' views of their role and efficacy in supporting young people's mental health. International Journal of Health Promotion and Education, 53(2), 102-114.

Moesch, K., Kenttä, G., Kleinert, J., Quignon-Fleuret, C., Cecil, S., \& Bertollo, M. (2018). FEPSAC position statement: Mental health disorders in elite athletes and models of service provision. Psychology of Sport and Exercise, 38, 61-71.

Moreland, J. J., Coxe, K. A., \& Yang, J., (2018). Collegiate athletes' mental health services utilization: A systematic review of conceptualizations, operationalizations, facilitators, and barriers. Journal of Sport and Health Sciences, 7(1), 58-69.

Morgan, D. L. 2008. Snowball sampling. The SAGE Encyclopedia of Qualitative Research Methods. http://sk.sagepub.com/reference/research/n425.xml.

Neal, T. L., Diamond, A. B., Goldman, S., Liedtka, K. D., Mathis, K., Morse, E. D., ... Welzant, V. (2015). Interassociation recommendations for developing a plan to recognize and refer student-athletes with psychological concerns at the secondary school level: A consensus statement. Journal of Athletic Training, 50, 231-249.

Orlinsky, D. E., Grave, K., \& Parks, B. K. (1994). Process and outcome in psychotherapy - noch einmal. In A. E. Bergin \& S. L. Garfield (Eds.), Handbook of psychotherapy and behaviour change (pp. 257-310). New York: Wiley.

Parham, W. D. (1993). The intercollegiate athlete: A 1990s profile. Counseling Psychologist, 21, 411-429. 
Peachey, D., Hicks, V., \& Adams, O. (2013). An imperative for change: Access to psychological services for Canada. Retrieved from http://www.cpa.ca/docs/File/Position/An_Imperative_for_Change.pdf.

Pedersen, E. R., \& Paves, A. P. (2014). Comparing perceived public stigma and personal stigma of mental health treatment seeking in a young adult sample. Psychiatry Research, 219, 143-150.

Petrie, T. A., Greenleaf, C., Reel, J., \& Carter, J. (2009). Personality and psychological factors as predictors of disordered eating among female collegiate athletes. Eating Disorders, 17(4), $302-321$.

Pinkerton, R. S., Hinz, L. D., \& Barrow, J. C. (1989). The college student-athlete: Psychological considerations and interventions. Journal of American College Health, 37, 218-226.

Powell, A. J., \& Myers, T. D. (2017). Developing mental toughness: Lessons from Paralympians. Frontiers in Psychology, 8, 1-16.

Public Health Agency of Canada (PHAC). (2006). The human face of mental health and mental illness in Canada 2006. Retrieved from http://www.phacaspc. gc.ca/publicat/humanhumain06/pdf/human_face_e.pdf.

Reardon, C. L., \& Factor, R. M. (2010). Sport psychiatry: a systematic review of diagnosis and medical treatment of mental illness in athletes. Sports Medicine, 40, 961-980.

Reardon, C. L., Hainline, B., Aron, C. M., Baron, D., Baum, A. L., Bindra, A., ... Engebretsen, L. (2019). Mental health in elite athletes: International Olympic Committee consensus statement. British Journal of Sports Medicine, 53, 667-699. 
Rice, S. M., Purcell, R., De Silva, S., Mawren, D., McGorry, P. D., \& Parker, A. G. (2016). The mental health of elite athletes: A narrative systematic review. Sports Medicine, 46(9), $1333-1353$.

Roe, M., Malone, S., Blake, C., Collins, K., Gissane, C., Büttner, F., ...Delahunt, E. (2017). A six stage operational framework for individualising injury risk management in sport. Injury Epidemiology, 4(26), 1-6.

Sandelowski, M., \& Leeman, J. (2012). Writing usable qualitative health research findings. Qualitative Health Research, 22, 1404-1413.

Sareen, J., Jagdeo, A., Cox, B. J., Clara, I., ten Have, M., Belik, S. L., ... Stein, M B. (2007). Perceived barriers to mental health service utilization in the United States, Ontario, and the Netherlands. Psychiatric Services, 58(3), 357-364.

Schaal, K., Tafflet, M., Nassif, H., Thibault, V., Pichard, C., Alcotte, M., ... Toussaint, J. (2011). Psychological balance in high level athletes: Gender-based differences and sport-specific patterns. PLoS One, 6, 1-8.

Sebbens, J., Hassmén, P., Crisp, D., \& Wensley, K. (2016). Mental health in sport (MHS): Improving the early intervention knowledge and confidence of elite sport staff. Frontiers in Psychology, 7(911), 1-9.

Sheard, M., Golby, J., \& van Wersch, A. (2009). Progress toward construct validation of the Sports Mental Toughness Questionnaire (SMTQ). European Journal of Psychological Assessment, 25, 186-193.

Smith, A. M., \& Milliner, E. K. (1987). Injured athletes and the risk of suicide. Journal of Athletic Training, 29, 337-341. 
Smith, B. (2015). Narrative analysis. In E. Lyons and A. Coyle (eds.) Analysing qualitative data in psychology (pp. 202-221). London, U. K.: Sage Publications.

Smith, D. M. (2005). In their own voices: Attitudes about mental health utilization by African American females at a predominantly White institution. (Unpublished doctoral dissertation). University of Tennessee, Knoxville.

Souter, G., Lewis, R., \& Serrant, L. (2018). Men, mental health and elite sport: A narrative review. Sports Medicine, 4(57), 1-8.

Vaismordi, M., Turunen, H., \& Bondas, T. (2013). Content analysis and thematic analysis: Implications for conducting a qualitative descriptive study. Nursing and Health Sciences, $15,398-405$.

Van Slingerland, K. J., Durand-Bush, N., Bradley, L., Goldfield, G., Archambault, R., Smith, D., ... Kenttä, G. (2019). Canadian Centre for Mental Health and Sport (CCMHS) position statement: Principles of mental health in competitive and high-performance sport. Clinical Journal of Sport Medicine, 29, 173-180.

Wahto, R. S., Swift, J. K., \& Whipple, J. L. (2016). The role of stigma and referral source in predicting college student-athletes' attitudes toward psychological help-seeking. Journal of Clinical Sport Psychology, 10, 85-98.

Watson, J. (2005). College student-athletes' attitudes toward help-seeking behavior and expectations of counseling services. Journal of College Student Development, 46, 442449.

Weigand, D. A., Richardson, P. A., \& Weinberg, R. S. (1999). A two-stage evaluation of a sport psychology internship. Journal of Sport Behavior, 22, 83-104. 
Wylleman, P., Alfermann, D., \& Lavallee, D. (2004). Career transitions in sport: European perspectives. Psychology of Sport and Exercise, 5, 7-20.

Yang, J., Peek-Asa, C., Corlette, J. D., Cheng, G., Foster, D. T., \& Albright, J. (2007).

Prevalence of and risk factors associated with symptoms of depression in competitive collegiate student athletes. Clinical Journal of Sport Medicine, 17, 481-487.

Yoon, J., Bae, M., Kang, H., \& Kim, T. (2018). Descriptive epidemiology of sports injury and illness during the Rio 2016 Olympic games: A prospective cohort study for the Korean team. International Journal of Sports Science and Coaching, 13(6), 939-946.

Yukelson, D. (2001). Immersion into inter-collegiate athletic environment: Benefits, risks and management. In A. Papaioannou, M. Goudas, \& Y. Theodorakis (Eds.), Proceedings of $10^{\text {th }}$ World Congress of Sport Psychology. Vol 3. In the dawn of the new millennium (pp. 224-226). Thessaloniki, Hellas: Christodoulidi Publications. 